\title{
2. CORRELATION OF QUATERNARY TEPHRAS THROUGHOUT THE IZU-BONIN AREAS ${ }^{1}$
}

\author{
Kantaro Fujioka, ${ }^{2}$ Akira Nishimura, ${ }^{3}$ Yoshiko Matsuo, ${ }^{4}$ and Kelvin S. Rodolfo ${ }^{5}$
}

\begin{abstract}
Quaternary marine tephras in the Izu-Bonin Arc offer significant information about explosive volcanic activities of the arc. Visual core descriptions, petrographic examinations, and chemical and grain-size analyses were conducted on tephras of backarc, arc, and forearc origin. Tephras are black and white and occur in simple and multiple modes with mixed and nonmixed ashes of black and white glass shards. The grain size distributions of the tephras are classified into three categories: coarse, white pumiceous, and fine white and black well-sorted types. The frequency of occurrence of the white and black tephras differs within the tectonic settings of the arc. Chemically, the Quaternary tephras in this region belong to low-alkali tholeiitic series with lower $\mathrm{K}_{2} \mathrm{O}$ and $\mathrm{TiO}_{2}$ than normal ordinary arc volcanic materials. Several tephras from different sites along the forearc correlate with each other and with tephras in the Shikoku Basin site and with Aogashima volcanics. These volcanic ashes resemble those in other backarc rifting areas, such as in the Fiji, Okinawa (Ryukyu), and Mariana regions.
\end{abstract}

\section{INTRODUCTION}

The distribution of Quaternary marine tephras around the Japanese Islands has been well documented by studies of piston core samples taken in the region (Machida and Arai, 1983, 1988; Furuta et al., 1986). However, tephras along the Izu-Bonin Arc are not as well studied, except for those of Oshima Island, south of Tokyo (Nakamura, 1964). Drilling along the Izu-Bonin Arc provides an excellent opportunity to document the chemistry and distribution of these opean-ocean tephras and to correlate them, if possible, to those around the Japanese Islands. Moreover, obtaining information on the volcanic ash layers along the Izu-Bonin Arc is of primary importance for understanding the tectonic evolution of the arc.

During Ocean Drilling Program (ODP) Leg 126, we recovered a large number of tephra layers from the Aogashima and Sumisujima area in the Izu-Bonin Arc south of Hachijojima Island, several hundred kilometers from Tokyo. Seven sites were drilled around the active volcanic islands of Aogashima and Sumisujima: three sites in the forearc basin (Sites 787, 792, and 793), two sites on the volcanic front (Sites 788 and 789), and two sites in the active backarc Sumisu Rift (Sites 790 and 791), resulting in a cross-sectional traverse of the arc through the forearc to the backarc (Figs. 1 and 2).

Site 787 in the Aogashima canyon, Site 792 at the fork of the Aogashima canyon, and Site 793 in the middle part of the upper slope basin were drilled in the forearc of the Izu-Bonin Arc (Fig. 1). The three sites have similar Oligocene to Holocene lithologies (Fig. 3). Three major lithologic units are evident: gravity flow, hemipelagic sediments, and volcaniclastic materials (Taylor, Fujioka, et al., 1990). The sedimentation rates of the three units at each of these three sites are also identical to each other: high in the gravity flow facies, very low in the hemipelagic facies, and moderately high in the volcaniclastic facies (Taylor, Fujioka, et al., 1990). More than 300 tephra layers were successfully recovered from these three sites.

Site 788 was drilled on the footwall of the Sumisu backarc rift, a well-studied backarc rift of the Izu-Bonin Arc (Fujioka, 1983a, 1983b,

\footnotetext{
'Taylor, B., Fujioka, K., et al., 1992. Proc. ODP, Sci. Results, 126: College Station, TX (Ocean Drilling Program).

${ }^{2}$ Ocean Research Institute, University of Tokyo, 1-15-1 Minamidai, Nakano, Tokyo 164, Japan (present address: Japan Marine Science and Technology Center, 2-14 Natsushima, Yokosuka, Kanagowa 238, Japan).

${ }^{3}$ Marine Geology Department, Geological Survey of Japan, 1-1-3 Yatabe, Tsukuba 305, Japan.

${ }_{4}^{4}$ Institute of Geosciences, Shizuoka University, 836 Oya, Shizuoka 422, Japan.

${ }^{5}$ Department of Geological Sciences, University of Illinois at Chicago, P.O. Box 4348 , Chicago, IL 60680, U.S.A.
}

1988; Brown and Taylor, 1988; Murakami, 1988; Nishimura and Murakami, 1988; Yamazaki, 1988; Ikeda and Yuasa, 1989; Fujioka et al., 1990) (Fig. 4). At this site, thick pumiceous layers were recovered with Pliocene altered pumice beds at the base.

Sites 790 and 791 were drilled $2.5 \mathrm{~km}$ apart in the eastern halfgraben of the eastern Sumisu Rift (Fig. 4). Three major lithologies, including basement basalts, were identified in these Sumisu Rift sites. The upper half of the section consists of coarse pumiceous beds with four or five cycles of coarse pumice units underlying thin nannofossil-rich hemipelagites. The basement of the rift basin was characterized by a frothy vesicular basalt similar in chemical composition to the backarc basin basalt (Gill et al., 1990; Taylor, Fujioka, et al., 1990; Leg 126 Shipboard Scientific Party, 1989; Leg 126 Scientific Drilling Party, 1989).

We recovered more than 500 volcanic ash layers and ashy intervals in the Quaternary sediments along the Izu-Bonin Arc during Leg 126. This report documents the chemical nature of these volcanic ash layers and the accumulation rate of the volcaniclastic materials in the region. We also summarize the features and timing of the explosive volcanism that took place along the Izu-Bonin Arc during the Quaternary.

\section{METHODS}

\section{Smear Slide Observations}

To determine the mineral assemblage, mode, and morphology of the glass shards and the nature of the lithic fragments of the volcanic ash layers, we made smear slides of each tephra unit following the procedure outlined in the explanatory notes of the Leg 126 Initial Reports volume (see Taylor, Fujioka, et al., 1990).

\section{Grain-size Analysis}

The grain-size distribution of the tephras were determined with a laser light grain-size analyzer for particles smaller than $500 \mu \mathrm{m}$; for those larger than $500 \mu \mathrm{m}$, the samples were first sieved, and then their weights were measured.

Materials finer than $44 \mu \mathrm{m}$ were eliminated by sieving. The 500 $\mu \mathrm{m}$ fraction was analyzed with an automatic laser-beam particle analyzer (Model SALD 1100 of Shimadzu Co Ltd., Japan). Sediments were put into a glass vessel with distilled water. After being mixed in an ultrasonic cleaner for $10 \mathrm{~s}$, the samples are put into the quartz cell of the laser unit. In this unit, laser light refracts suspended materials, and the ratio of the reflected light from particles inside the quartz cell are measured by a photodetector. The accuracy of the grain-size distribution for each tephra layer is more than $95 \%$. 


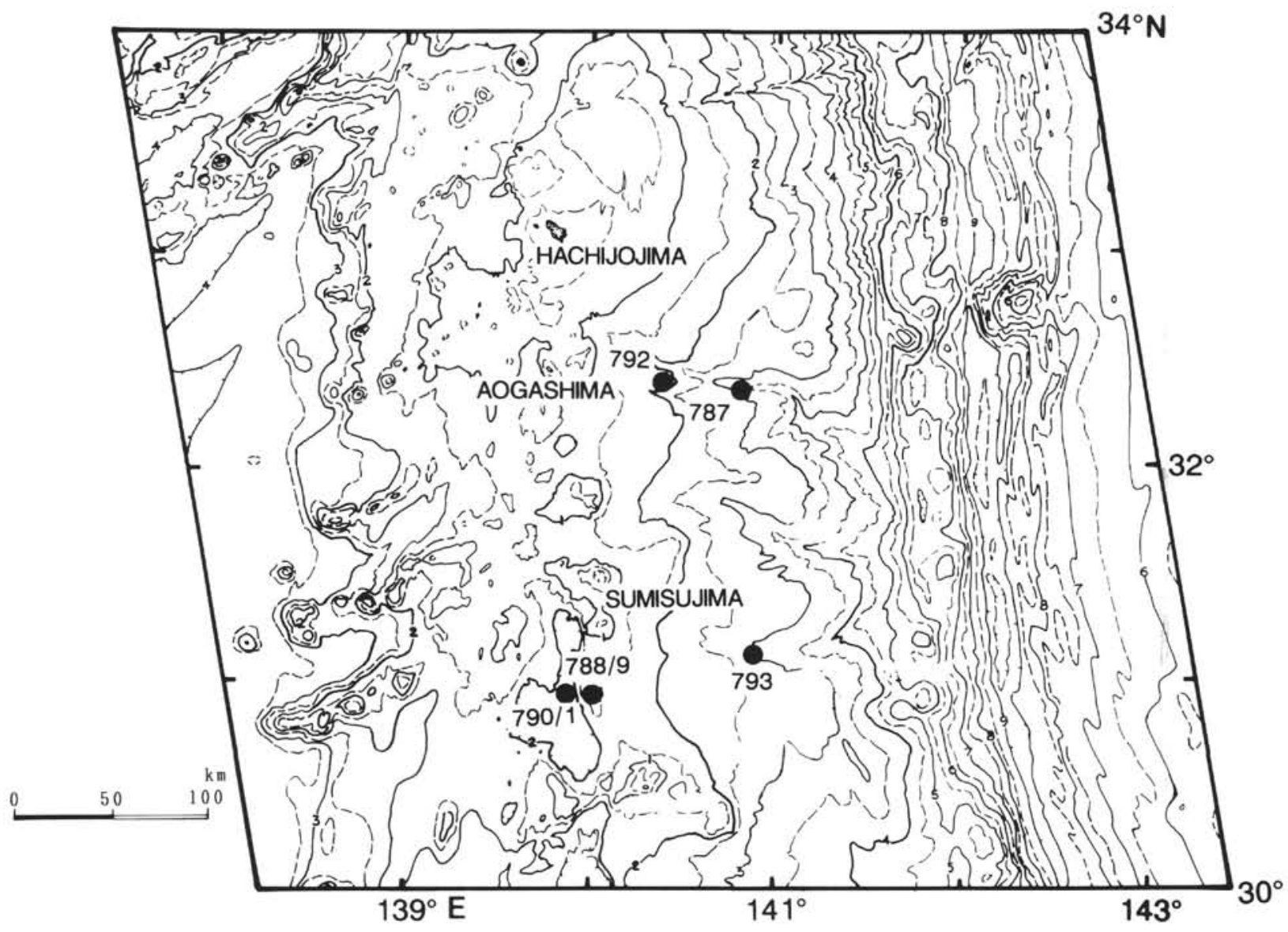

Figure 1. Bathymetric map of the Izu-Bonin Arc-trench system between $30^{\circ} \mathrm{N}$ and $34^{\circ} \mathrm{N}$ and locations of the drilling sites (solid circles) of Leg 126 . Contour interval $=500 \mathrm{~m}$ (numbers on contour lines indicate depth in $\mathrm{km}$ ).

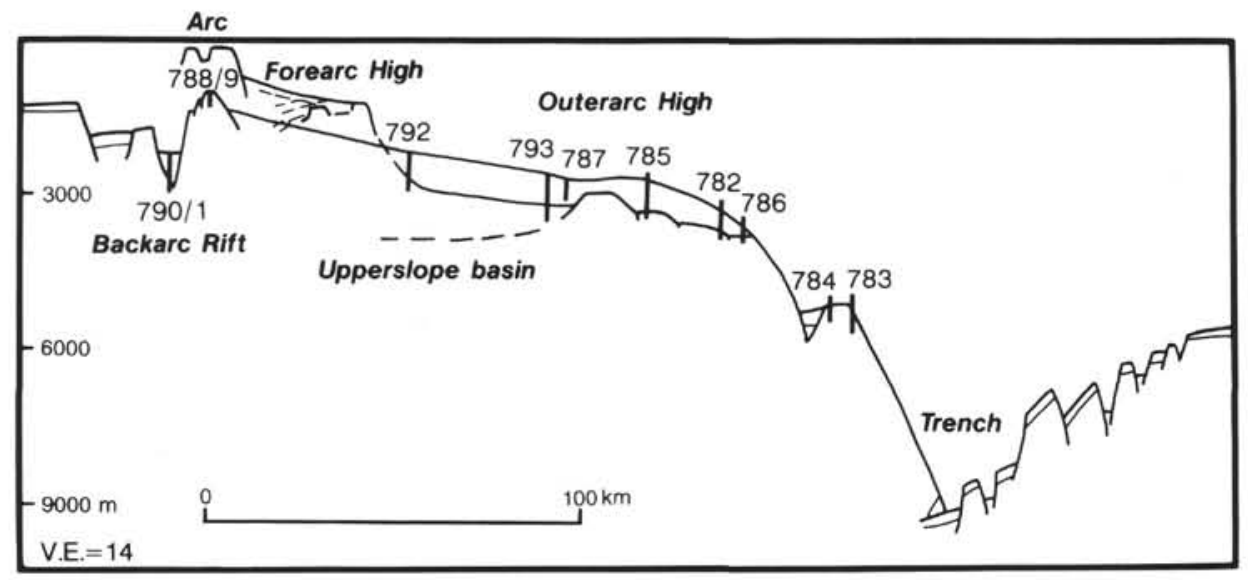

Figure 2. Schematic cross section of the Izu-Bonin Arc with drilling sites. V.E. = vertical exaggeration.

\section{Refractive Indices of Glass}

The refractive indices of glass shards from the volcanic ash layers were determined using the automatic refractive index measurements apparatus of Kyoto Fission Track Co. Ltd., Model RIMS-86 following the procedures outlined in Yokoyama et al. (1986). In this proce- dure, the refraction index value follows the temperature change of the immersion liquid, which is automatically controlled by the instrument's thermomodule. When the glass flakes in the immersion liquid disappear under the microscope, the operator sends a signal to the microcomputer. After the procedural steps are repeated two times (for ascending and descending temperatures) on a glass flake, the micro- 


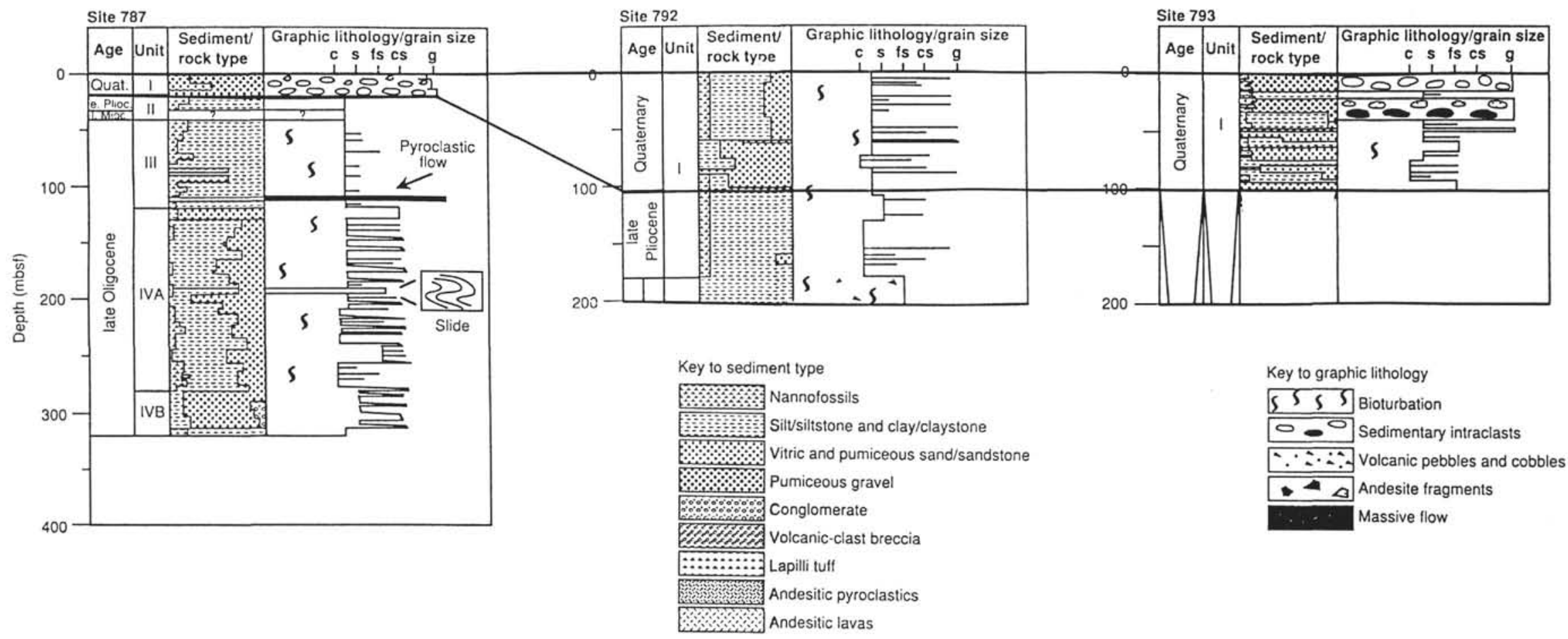

Figure 3. Lithostratigraphic diagram of Izu-Bonin forearc Sites 787, 792, and 793. Solid line indicates the bottom of the Quaternary sediments cored during Leg 126. 

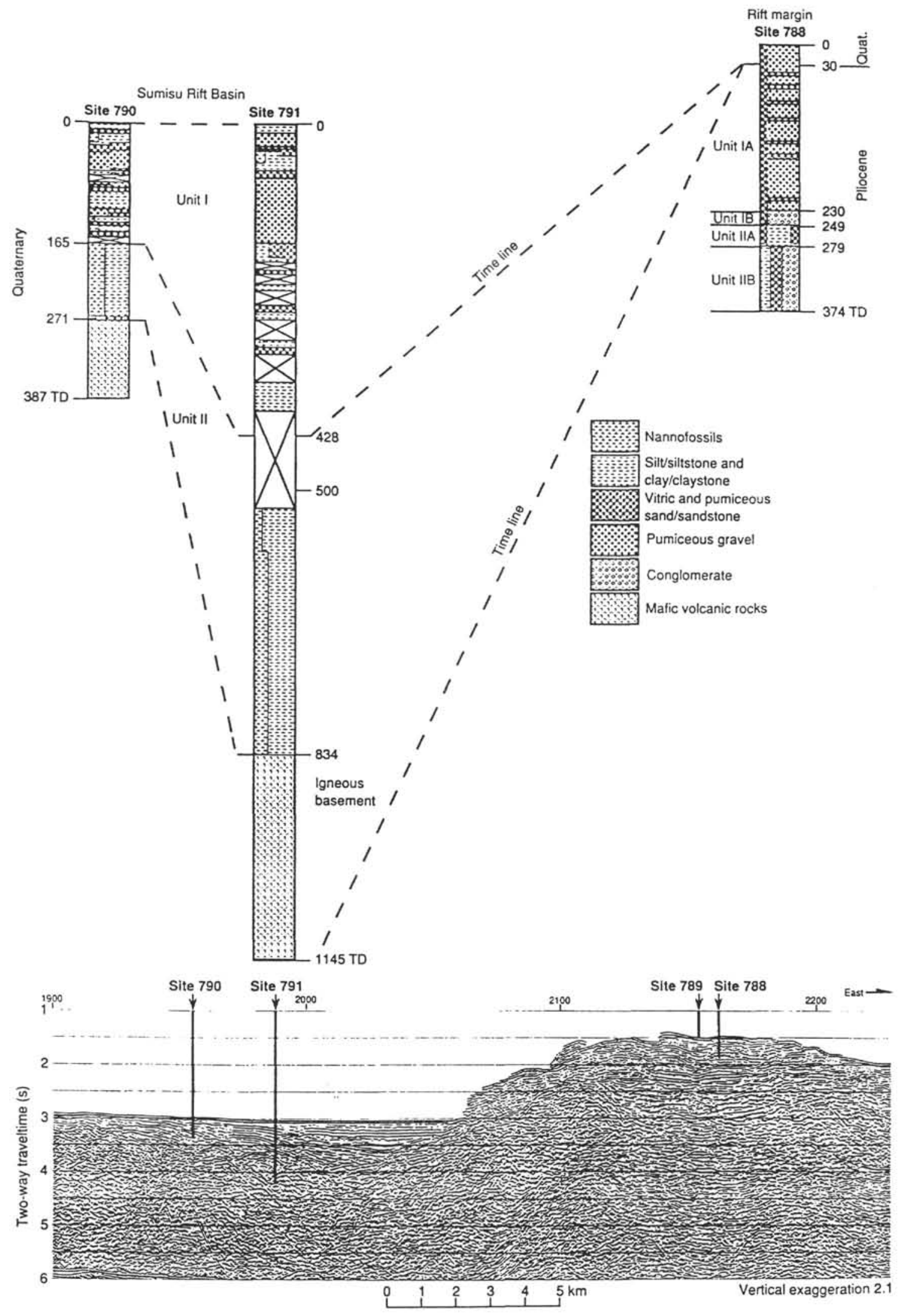

Figure 4. Lithostratigraphic diagram of backarc Sites $790 / 791$ and arc Site 788 (after correlation paper in Taylor, Fujioka, et al., 1990). Upper sketch is the stratigraphic correlation of the backarc sites; lower sketch is the seismic profile of the MCS Line Bon II-4 across the eastern footwall uplift and eastern half-graben of the Sumisu Rift. 
computer calculates the mean and standard deviation of the refractive index. The accuracy of the refractive index value of a single glass flake is $>99 \%$.

\section{Chemical Analyses with the Electron Probe Microanalyzer}

An electron probe microanalyzer was used to determine the percentage of 12 major elements of the glass shards as well as the minerals in the tephra layers. The tephras were cleaned with an ultrasonic cleaner in distilled water to eliminate clay fractions. Dried samples were impregnated on acrylic plates with 5-mm punches of epoxy resin, and polished thin sections of the volcanic ash layers were prepared. The experimental conditions for analyzing the glass shards were as follows: $15 \mathrm{kV}, 1.2 \times$ $10^{-7} \AA$, beam diameter of $10 \mu \mathrm{m}, 10$-s count for every oxide. Oxides and a single mineral were used as a standard for each oxide. The accuracy of each oxide ranges from $90 \%$ to $98 \%$, depending on the element. See Fujioka et al. (1980) and Furuta and Arai (1980a, 1980b) for details of the microprobe procedures.

\section{VISUAL OBSERVATIONS OF VOLCANIC ASH LAYERS}

Visual core descriptions and smear slide and binocular observations of the volcanic ash layers were conducted on board the JOIDES Resolution and during shore-based studies on the characterization of each ash layer. A list of all the tephras analyzed is given in Table 1. The colors of the tephras are white, gray, green, and black, depending on the chemistry of the glass shards, lithic fragments, and minerals. The glassy tephras are silt-sized, and the scoria and pumice range from sand to granule in size. The thickness of the volcanic ash layers ranges from several tens of centimeters (maximum $=146 \mathrm{~cm}$ ) to a few millimeters, depending on their distance from the source areas and the degree of bioturbation caused by postdepositional processes. Almost all the tephras are less than $10 \mathrm{~cm}$ thick, but tephras several tens of centimeters $(60 \mathrm{~cm})$ often occur in turbidites having scoriae and pumices. These thicker layers may be resedimented mixed layers. Sedimentary structures such as laminae, bioturbation, and grading were also observed.

The volcanic glasses recovered during Leg 126 occur as discrete layers and small pods or pockets or are dispersed in the muddy matrices. Some tephra layers have distinct grading structures, some are mixed with rhyolitic and basaltic material, and some are resedimented. Strong burrow structures caused by bottom-dwelling organisms, such as Zoophycos, Chondrites, and Planolites (Taylor, Fujioka, et al., 1990) also occur in the tephra layers, but these are rare.

Visually, the tephras are classified into two types: simple layer and multiple layer. They also can be classified into mixed and unmixed. The simple layer has a definite boundary in a mud matrix and consists of black and white tephras mixed to form a discrete layer. The multiple layer consists of sets of tephras that have not been diluted by the mud matrices and that have sharp contacts from layer to layer. The existence of the sharp boundaries in the multiple-layer volcanic ash layers suggests that explosive volcanism may take place regularly, with short volcanic quiescent periods intervening in which little or no mud matrix accumulates.

Two types of dark-colored volcanic ash layers were frequently encountered: (1) black, coarse (scoriaceous) and (2) black, fine, well sorted. Two types of light-colored volcanic ash layers were also frequently encountered during visual core observation: (1) white, coarse (pumiceous) and (2) fine, white. These four visually distinct volcanic ashes suggest that their origins are different from each other.

\section{Smear Slide and Binocular Observations}

Smear slide descriptions of all the volcanic ash layers sampled are presented in Table 2. Characteristic modes of occurrences of the volcanic glass shards are classified into three major types: translucent, black, and mixed. The translucent type contains more than $85 \%$ glass shards with no lithic fragments. The black type has a few lithic fragments and $15 \%-40 \%$ crystals. The mixed type contains both black and translucent volcanic glass shards. The shapes of the volcanic glass shards are bubble wall (bw), tabular (tub), and pumice (pm) for the translucent type and brown-colored, bubble-wall (brbw) and crystalrich type for the black ashes. Common mineral assemblages for all the volcanic ash layers identified under the petrographic and binocular microscopes are plagioclase, clinopyroxene, and opaque minerals. Orthopyroxene and biotite are rarely encountered. Hornblendes are present but rare in the white ash and crystal-rich black ashes.

\section{ACCUMULATION RATES OF THE VOLCANIC ASH LAYERS}

To calculate the accumulation rates of the volcaniclastic materials, we assumed that the input of volcaniclastic material was instantaneous as compared with the hemipelagic muds. Figures 5,6 , and 7 illustrate the accumulation rate of the tephras obtained this way. The accumulation rates of the ash at backarc Sites 790 and 791 show a distinct maximum since $0.2 \mathrm{Ma}$. The accumulation rate of the volcaniclastic materials of the forearc sites (Figs. 5 and 6) shows two maxima, one around $1.0-0.6 \mathrm{Ma}$ and another at $0.2-0.4 \mathrm{Ma}$. The older peaks include the rapid accumulation of the dark-colored ashes (i.e., the basaltic ashes). However, the younger peak consisting mostly of rhyolitic ash layers has a much higher accumulation rate. The accumulation rate of the forearc region is quite similar in its pattern to those obtained in the ashes distributed in the south Boso Peninsula (Kotake, 1988).

\section{GRAIN-SIZE DISTRIBUTION OF THE VOLCANIC ASH LAYERS}

The grain size of the volcanic ash layers contains information about (1) the origin of the ash, (2) the source region of the tephra, and (3) the depositional mechanism of tephras. The grain-size distribution of the volcanic ash layers of the forearc sites are classified into three types of distribution patterns (Figs. 8A and $8 \mathrm{~B}$ and Table 3). The first type is characterized by white, coarse ash layers with a median diameter around $3 \phi$ (Inmann's $\phi$ scale; Inmann, 1952) and a wellsorted cumulative curve; skewness is small. Examples of this type of grain-size distribution include Samples 126-792A-4H-4, 96-98 $\mathrm{cm}$, and 126-792B-19H-2, 36-38 cm. The second characteristic distribution is a black ash that has a well-sorted cumulative curve with a $3-4 \phi$ median diameter; skewness is small, but kurtosis is high. Examples of this type of grain-size distribution include Samples 126-792A-3H-5, 78-80 cm, and 126-793A-3H-2, 50-52 $\mathrm{cm}$. The third characteristic grain-size distribution is typified by a fine white ash layer, either well or poorly sorted, with a median diameter of $4 \phi$. The poorly sorted tephras are skewed toward the finer portions, whereas skewness is small in the well-sorted portion. Examples of the third grain-size distribution type include Samples $126-788 \mathrm{C}-11 \mathrm{H}-4,108-110 \mathrm{~cm}, 126-792 \mathrm{~A}-1 \mathrm{H}-5,81-83$ $\mathrm{cm}$, and 126-793A-3H-2, 69-71 cm. Tephras with well-sorted, fine median diameters may be the result of fallout deposits, whereas those skewed toward a finer grain size may be the result of subaqueous volcanism (Sparks et al., 1981; Walker, 1971).

The tephra grain-size distribution suggests that distribution types 1 and 2 represent input from a nearby source (i.e., the Izu-Bonin Arc), but the black tephras with low kurtosis may come from secondary processes. The third type of tephra grain-size distribution may represent input from the western part of the Philippine Sea.

The backarc tephras are classified into two types of grain-size distribution curves: coarse pumiceous and fine glass. Characteristic features of the coarse tephras include a broad size distribution ranging from clay to granule size (Nishimura et al., this volume); medium 
Table 1. Volcanic ash layers at Site 790.

\begin{tabular}{|c|c|c|c|}
\hline $\begin{array}{l}\text { Layer } \\
\text { no. }\end{array}$ & $\begin{array}{l}\text { Core, section, } \\
\text { interval }(\mathrm{cm})\end{array}$ & $\begin{array}{l}\text { Thickness } \\
(\mathrm{cm})\end{array}$ & Description and remarks \\
\hline \multicolumn{4}{|l|}{ Hole 790B } \\
\hline 1 & $\underset{2 \mathrm{H}-2}{1 \mathrm{H}-1,14(\text { top })-140 \text { (base) }}$ & 726 & Thick pumice layer \\
\hline 2 & $\begin{array}{l}2 \mathrm{H}-2,145-150^{*} \\
2 \mathrm{H}-3,{ }^{*} 0-5\end{array}$ & $5 / 3 / 2$ & Very dark gray $(5 Y 3 / 1) /$ brownish black(5YR2/I/grayish black(N2): silt/silt/v.f.sand \\
\hline 3 & $2 \mathrm{H}-3,7-11$ & 4 & Brownish black $(5 Y R 2 / 1)$; silt \\
\hline 4 & $2 \mathrm{H}-3,17-20$ & 3 & Brownish black $(5 Y R 2 / 1)$; silt \\
\hline 5 & $2 \mathrm{H}-3,39-46$ & 7 & Grayish black(N2)/brownish black(5YR2/1); silt \\
\hline 6 & $\begin{array}{l}2 \mathrm{H}-3,148-150 * \\
2 \mathrm{H}-4, * 0-18\end{array}$ & 20 & Grayish black(N2): silt (laminated at lowest $5 \mathrm{~cm}$ ) \\
\hline 7 & $2 \mathrm{H}-4,61-74$ & 3 & Light olive gray $(5 Y 6 / 1)$ : silt \\
\hline 8 & $\begin{array}{l}2 \mathrm{H}-4,127-150 * \\
2 \mathrm{H}-5, * 0-138\end{array}$ & $76 / 85$ & Light olive gray $(5$ Y $4 / 1)$ : silt/v.f s-m.s(grading) \\
\hline 9 & $2 \mathrm{H}-6,42-77$ & 35 & Brownish black $(5 Y R 2 / 1)$; silt \\
\hline 10 & $3 \mathrm{H}-1,27-56$ & 29 & Grayish black(N2): silt \\
\hline 11 & $\begin{array}{l}3 \mathrm{H}-1,70-150^{*} \\
3 \mathrm{H}-2, * 0-29\end{array}$ & $44 / 65$ & Grayish black(N2); silt/f.-m.sand(laminated) \\
\hline 12 & $3 \mathrm{H}-2,58-150^{*}$ & $152 \pi 7$ & $\begin{array}{l}\text { Olive gray }(5 Y 5 / 2) / \text { olive gray }(5 Y 4 / 2) / \text { dark olive gray }(5 Y 3 / 2) \text {; } \\
\text { silt/silt-m.sand(grading) }\end{array}$ \\
\hline 13 & $\begin{array}{l}3 \mathrm{H}-3, * 0-137 \\
3 \mathrm{H}-4,29 \text { (top)-7(base) }\end{array}$ & 3628 & Thick pumice layer II \\
\hline 14 & $7 \mathrm{H}-3,27-31$ & 4 & Brownish black $(5 Y R 2 / 1)$; silt \\
\hline 15 & $7 \mathrm{H}-3,63-66$ & 3 & Olive black $(5 Y 2 / 1)$; silt \\
\hline 16 & $7 \mathrm{H}-3,85-93$ & $3 / 3 / 2$ & Olive gray $(5 Y 4 / 1) /$ olive gray $(5 Y 4 / 1)$ : silt/silt/silt \\
\hline \multirow[t]{2}{*}{17} & $7 \mathrm{H}-3,115-150^{*}$ & $50 / 551$ & $\begin{array}{l}\text { Gray }(5 \mathrm{Y} 5 / 1) / \text { olive gray }(5 \mathrm{Y} 4 / 2) \text { olive gray }(5 \mathrm{Y} 4 / 1) /(5 \mathrm{Y} 4 / 1) / \text { olive } \\
\text { gray }(5 \mathrm{Y} 4 / 2) /(5 \mathrm{Y} 4 / 2) /(5 \mathrm{Y} 4 / 2) \text { s silt/silt/v. f sand/v. f. sand } / \mathrm{f} \text {.sand }\end{array}$ \\
\hline & $\begin{array}{l}7 \mathrm{H}-4, * 0-150 * * \\
7 \mathrm{H}-5, * * 0-150 * * * \\
7 \mathrm{H}-6, * * * 0-130\end{array}$ & $\begin{array}{l}230 / 45 / \\
35 / 50\end{array}$ & \\
\hline \multirow{3}{*}{19} & $7 \mathrm{H}-6,143-147$ & 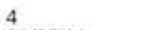 & Reddish gray (10R6/1); silt \\
\hline & $8 \mathrm{H}-1,+0-150 *+$ & $90 / 25 / 11$ & Light gray $(5 Y 7 / 1) / g r a y(5 Y 5 / 1) /(5 Y 5 / 1) /$ olive \\
\hline & & & $\begin{array}{l}\text { gray }(5 \mathrm{Y} 4 / 1) /(5 \mathrm{Y} 5 / 1) /(5 \mathrm{Y} 4 / 1) \text { dark olive gray }(5 \mathrm{Y} 3 / 2) / \text { olive black }(5 \mathrm{Y} 2 / 1 \mathrm{f} \text { : } \\
\text { pumice granule (maximum diameter }=3.5 \mathrm{~cm}) / \text { mixture of pumice pebble } \\
\text { and silt/silt/silt/silt/silt/v.f.sand/f.sand }\end{array}$ \\
\hline \multirow{3}{*}{20} & $\begin{array}{l}8 \mathrm{H} 2, * 0-90 \\
8 \mathrm{H}-2,106-150^{*}\end{array}$ & $\begin{array}{l}11 / 23 / 70 / 17 / 3 \\
39 / 1 / 40\end{array}$ & Brownish black(5YR2/1)/grayish black(N2)/olive \\
\hline & & & $\begin{array}{l}\text { black }(5 Y 2 / 1) /(\mathrm{N} 2) /(\mathrm{N} 2) / \text { dark olive gray }(5 \mathrm{Y} 3 / 2) \text {; silt/v.f.sand/silt with } \\
\text { grayish black } v . \mathrm{f} \text {.sand thin layers/v.f.sand/f.sand/v.f.sand }\end{array}$ \\
\hline & $8 \mathrm{H}-3,{ }^{*} 0-85$ & /9/32/8 & \\
\hline 21 & $8 \mathrm{H}-3,143-148$ & & Dark olive gray $(5 Y 3 / 2) ;$ v.f. sand \\
\hline 22 & $8 \mathrm{H}-4,0-38$ & $35 / 3$ & Olive black $(5 Y 2 / 1)$; silt/f.sand(laminated) \\
\hline 23 & $8 \mathrm{H} 4,48-62$ & $10 / 2 / 2$ & Olive black $(5 Y 2 / 1) / g r a y i s h$ black(N2)/olive black $(5 Y 2 / 1)$, silt/v.f.sand/silt \\
\hline 24 & $8 \mathrm{H}-4,115-150^{*}$ & $26 / 18$ & $\begin{array}{l}\text { Olive black }(5 \mathrm{Y} 2 / 1) / \text { grayish black }(\mathrm{N} 2) \text {; silt with v.f.sand } \\
\text { laminated/v.f.-f.sand(grading) }\end{array}$ \\
\hline 25 & $\begin{array}{l}8 \mathrm{H}-5, * 0-9 \\
8 \mathrm{H}-5,38-131\end{array}$ & $11 / 41 / 41$ & $\begin{array}{l}\text { Brownish black }(5 Y R 2 / 1) / \text { olive black }(5 Y 2 / 1) / g r a y i s h ~ b l a c k(N 2) \text { : f.sand with } \\
\text { pumice m.sand laminated/silt with grayish black(N2) v.f.sand thin } \\
\text { layers/v.f.-m.sand(grading) }\end{array}$ \\
\hline 26 & $8 \mathrm{H}-6,14-17$ & 3 & Dark olive gray $(5 Y 3 / 2)$; silt \\
\hline 27 & $8 \mathrm{H}-6,33-35$ & 2 & Dark olive gray $(5 Y 3 / 2)$; silt \\
\hline 28 & $8 \mathrm{H}-6,54-63$ & & Gray $(5 Y 5 / 1)$; silt \\
\hline 29 & $8 \mathrm{H}-6,74-92$ & $3 / 2 / 7 / 2 / 2$ & $\begin{array}{l}\text { Olive black(5Y2/1)/grayish black(N2)/olive black(5Y2/1)/grayish } \\
\text { black(N2)/olive black(5Y2/1); silt/v.f.sand/silt/v.f.sand/silt }\end{array}$ \\
\hline 30 & $\begin{array}{l}8 \mathrm{H}-7,43 \text { (top)-15(base) } \\
10 \mathrm{H}-5 \text {. }\end{array}$ & 1622 & Thick pumice layer III \\
\hline \multirow{10}{*}{$\begin{array}{l}31 \\
32\end{array}$} & $10 \mathrm{H}-5,22-24$ & 2 & Brownish black $(5 Y R 2 / 1)$; silt \\
\hline & $10 \mathrm{H}-5,32-150^{*}$ & & Olive gray $(5 Y 4 / 1) /$ dark greenish gray $(5 G Y 4 / 1) /(5 Y 4 / 1) /$ very dark \\
\hline & & & $\begin{array}{l}\text { gray }(5 Y 4 / 2) /(5 Y 4 / 1) /(5 Y 4 / 2) /(5 Y 4 / 1) /(5 Y 4 / 2) / \text { dark olive } \\
\text { gray }(5 Y) /(5 Y) /(5)\end{array}$ \\
\hline & & & gray $(5 \mathrm{Y} 3 / 2) /(5 \mathrm{Y} 3 / 2) /$ gray $(5 \mathrm{Y} 5 / 1) /$ olive black $(5 \mathrm{Y} 2 / 1) /(5 \mathrm{Y} 3 / 2)$; silty \\
\hline & & & clay/silty clay/silt/silt/silt/v.f.sand/silty v.f. \\
\hline & & & sand/silt/v.f.sand/silt/c.silt/v.f.sand/f.sand/f.sand/m.sand \\
\hline & $10 \mathrm{H}-6,{ }^{*} 0-146$ & $5 / 5 / 83 / 2 / 23 / 8 /$ & \\
\hline & & $1 / 4 / 5 / 6 / 4$ & \\
\hline & & $4 / 34 / 14 / 2$ & \\
\hline & & $/ 8 / 20$ & \\
\hline \multicolumn{4}{|l|}{ Hole $790 \mathrm{C}$ - } \\
\hline & $1 \mathrm{H}-4,70-72$ & 2 & Olive gray $(5 Y 4 / 1)$; v.f.sand \\
\hline 34 & $\begin{array}{l}1 \mathrm{H}-4,115 \text { (top) }-43 \text { (base) } \\
5 \mathrm{H}-3\end{array}$ & 3628 & Thick pumice layer IV \\
\hline 35 & $5 \mathrm{H}-3,55-59$ & 4 & Olive $(5 Y 5 / 4)$; f sand \\
\hline 36 & $5 \mathrm{H}-3.67-70$ & 3 & Olive $(5 Y 5 / 4) ;$ f.sand \\
\hline 37 & $5 \mathrm{H}-3,87$ & $<1$ & Dusky green $(5 \mathrm{G} 3 / 2)$; silt \\
\hline 38 & $5 \mathrm{H}-3,91-111$ & 20 & Grayish black(N2); clayey silt-f.s(grading) \\
\hline 39 & $5 \mathrm{H}-3,111-150^{*}$ & $39 / 60$ & Grayish black(N2)/dark gray(N3); silt/c.sand(grading) \\
\hline 40 & $5 \mathrm{H}-4,96-136$ & $26 / 14$ & Dark gray $(\mathrm{N} 3) /$ black $(\mathrm{N} 1)$; clayey silt $/ \mathrm{m}$.sand \\
\hline 41 & $5 \mathrm{H}-5,6-15$ & & Dark gray(N3): silt \\
\hline 42 & $5 \mathrm{H}-5,20-35$ & 15 & Dark gray(N3); clayey silt/v.f.sand \\
\hline 43 & $5 \mathrm{H}-5,43-95$ & $49 / 3$ & Dark gray(N3)/grayish black(N2); clayey silt/m.sand \\
\hline 44 & $5 \mathrm{H}-6,6-21$ & 15 & Medium dark gray(N4); silt-v.f.sand(grading) \\
\hline 45 & $5 \mathrm{H}-6,62-72$ & 10 & Medium dark gray(N4); silt-v.f.sand(grading) \\
\hline 46 & $5 \mathrm{H}-6,82$ & $<1$ & Dusky green $(5 \mathrm{G} 3 / 2)$; silt \\
\hline 47 & $5 \mathrm{H}-6,85$ & & Dusky green $(5 \mathrm{G} 2 / 1)$; silt \\
\hline \multirow{3}{*}{48} & $5 \mathrm{H}-6,120-150$ & $++2 / 28+$ & Medium light gray(N6)/olive gray $(5 Y 4 / 1)$ : silt/silt \\
\hline & $6 \mathrm{H}-1,+0-150^{*}$ & $++150 / 31$ & Olive gray $(5 Y 4 / 1) /$ olive gray $(5 Y 4 / 1) ;$ f.-m.sand/pumiceous \\
\hline & & & m.-c.sand(grading)(laminated) \\
\hline & $\begin{array}{l}6 \mathrm{H}-2, * 0-31 \\
6 \mathrm{H}-2,47-65\end{array}$ & & Light olive gray (5Y $6 / 1)$ : f sand(eradine) \\
\hline 51 & $6 \mathrm{H}-2,80-144$ & $\begin{array}{l}18 \\
57 \pi\end{array}$ & $\begin{array}{l}\text { Light olive gray }(5 \mathrm{Y} 6 / 1) ; \text { f.sand(grading) } \\
\text { Light olive gray }(5 \mathrm{Y} 6 / 1) \text { dark gray }(\mathrm{N} 3) \text {; silty clay/m.sand }\end{array}$ \\
\hline 52 & $6 \mathrm{H}-3.34-135$ & $29 / 73$ & Light olive gray(5Y6/1)/medium gray(N5); silt/m.sand(grading) (pumice \\
\hline 53 & $6 \mathrm{H}-4,5-51$ & $5 / 25 / 16$ & $\begin{array}{l}\text { maximum diameter }=1 \mathrm{~mm}) \\
\text { Light olive gray }(5 \mathrm{Y} 6 / 1) / \text { light olive gray }(5 \mathrm{Y} 6 / 1) / \text { dark gray }(\mathrm{N} 3) \text {; f.s/silty } \\
\text { clay/m.sand }\end{array}$ \\
\hline
\end{tabular}


Table 1 (continued).

\begin{tabular}{|c|c|c|c|}
\hline $\begin{array}{l}\text { Layer } \\
\text { no. }\end{array}$ & $\begin{array}{l}\text { Core, section, } \\
\text { interval }(\mathrm{cm})\end{array}$ & $\begin{array}{l}\text { Thickness } \\
\text { (cm) }\end{array}$ & Description and remarks \\
\hline 54 & $6 \mathrm{H}-4,60-8 \mathrm{I}$ & $11 / 10$ & $\begin{array}{l}\text { Light olive gray }(5 \mathrm{Y} 6 / 1) / \text { dark gray }(\mathrm{N} 3) \text {; silty clay with scattered pumice/f.sand } \\
\text { with an erosive base }\end{array}$ \\
\hline 55 & $6 \mathrm{H}-4,99-140$ & $2 / 23 / 16$ & $\begin{array}{l}\text { Olive gray }(5 Y 4 / 1) \text { olive gray }(5 Y 4 / 1) / \text { dark gray(N3); silty v.f.sand/silty } \\
\text { clay/m.sand }\end{array}$ \\
\hline 56 & $6 \mathrm{H}-5,7-77$ & $52 / 18$ & Light olive gray $(5 Y 6 / 1) /$ dark gray(N3); clayey silt/f.sand (laminated) \\
\hline 57 & $6 \mathrm{H}-5,96-118$ & $14 / 8$ & Light olive gray $(5 \mathrm{Y} 6 / 1) /$ dark gray(N3); clayey silt/f.sand (laminated) \\
\hline 58 & $\begin{array}{l}6 \mathrm{H}-5,141-150^{*} \\
6 \mathrm{H}-6,{ }^{*} 0-37\end{array}$ & $29 / 17$ & Light olive gray(5Y6/1)/dark gray(N3); clayey silt/silty v.f.sand \\
\hline $\begin{array}{l}59 \\
60\end{array}$ & $\begin{array}{l}6 \mathrm{H}-6,41-47 \\
6 \mathrm{H}-6,73-142\end{array}$ & 6 & $\begin{array}{l}\text { Light olive gray }(5 \mathrm{Y} 6 / 1) \text { : v.f.sand } \\
\text { Light olive gray }(5 \mathrm{Y} 6 / 1) / \text { /ight olive gray }(5 \mathrm{Y} 6 / 1) / \text { dark gray(N3); silty clay/silty }\end{array}$ \\
\hline 61 & $\begin{array}{l}7 \mathrm{H}-1,+0 \text { (top)-18(base) } \\
8 \mathrm{H}-\mathrm{CC} \text {. }\end{array}$ & $++1250+$ & $\begin{array}{l}\text { f.sand/f.sand } \\
\text { Thick pumice layer } V\end{array}$ \\
\hline 62 & $11 X-1,25$ & $<1$ & Silt \\
\hline 63 & $11 X-2,10-16$ & 6 & Very light gray(N8); clayey silt-v.f.sand(grading) \\
\hline 64 & $11 X-2,45-50$ & 5 & (10Y6/2); silt(grading) \\
\hline 65 & iix $-3,17-30$ & $12 / 1$ & Olive gray $(5 Y 5 / 2) /$ very light gray $(N 8)$; clayey silt-v.f.sand(grading)/clayey silt \\
\hline 66 & $11 \mathrm{X}-3,109-110$ & & Very light gray(N8); silt \\
\hline 67 & Iix-4, $32-47$ & $10 / 5$ & Olive gray $(5 Y 5 / 2) /$ dark gray(N3); clayey silt/c.sand \\
\hline 68 & IIX-4. $70-72$ & & Medium light gray(N6); v.f.sand \\
\hline $\begin{array}{l}69 \\
70\end{array}$ & $\begin{array}{l}11 X-4,110-120 \\
11 X-4,139-150^{\circ}\end{array}$ & $\begin{array}{l}10 \\
15 / 4 / 3 / 3\end{array}$ & $\begin{array}{l}\text { Dark gray(N3); silt(grading) } \\
\text { Olive gray(5Y5/2)/olive gray ( } 5 \text { Y } 5 / 2 \# / \text { light gray }(\mathrm{N} 7) \text { /medium dark gray }(\mathrm{N} 4) \text {; }\end{array}$ \\
\hline & & & silt/m.sand/silt/v.f.sand with and erosive base \\
\hline 71 & $12 X-1,5-14$ & 9 & Olive gray $(5 Y 4 / 1)$; silt \\
\hline 72 & $12 \times-1,20-23$ & 3 & Olive gray $(5 Y 4 / 1)$; silt \\
\hline 73 & $\begin{array}{l}12 X-1,26-28 \\
12 X-1,35-37\end{array}$ & $\frac{2}{2}$ & $\begin{array}{l}\text { Olive gray }(5 Y 4 / 1) ; \text { silt } \\
\text { Olive gray }(5 Y 4 / 1) \text {; silt }\end{array}$ \\
\hline 75 & $12 \mathrm{X}-1,40-42$ & 2 & Olive gray $(5 Y 4 / 1)$; silt \\
\hline \multirow[t]{2}{*}{76} & $12 X-1,45-69$ & $5 / 4 / 6 / 4 / 5$ & Olive gray $(5 Y 4 / 2) /$ olive gray $(5 Y 4 / 1) /$ olive gray $(5 Y 4 / 2) /$ olive \\
\hline & & & $\begin{array}{l}\text { black(SY } 2 / 1) / \text { olive black }(5 Y 2 / 1) \text {; silt/silt/m.sand with pumice } \\
\text { c.sand/f.sand/silt }\end{array}$ \\
\hline 77 & $12 \mathrm{X}-1.71-76$ & 5 & Olive black $(5 \mathrm{Y} 2 / 1) ; \mathrm{f}$.sand \\
\hline 78 & $\begin{array}{l}12 \mathrm{X}-1,130-140^{*} \\
12 \mathrm{X}-\mathrm{CC}, * 0-33\end{array}$ & $43+$ & Light olive gray $(5 Y 4 / 1)$; silt \\
\hline 79 & $13 \times-1,18-44$ & $7 / 5 / 4$ & Gray $(5 Y 5 / 1) /$ olive gray $(5 Y 4 / 1) /$ olive black $(5 Y 2 / 1)$; clayey silt/silt/f.sand \\
\hline 80 & $13 \mathrm{X}-1,50-52$ & & Olive gray $(5 Y 4 / 2)$; silt \\
\hline 81 & $13 \times-1,66-68$ & 2 & Olive black $(5 Y 2 / 1)$; silt \\
\hline 82 & $13 X-1.71-72$ & i & Olive black $(5 Y 2 / 1)$; silt \\
\hline 83 & $13 \mathrm{X}-1,79$ & $<1$ & Olive black $(5$ Y $2 / 1)$; silt \\
\hline 84 & $13 \times-1.81$ & $<1$ & Olive black $(5$ Y $2 / 1)$; silt \\
\hline 85 & $13 X-2.15-55$ & $31 / 9$ & Gray(5Y5/1)/gray(5Y $5 / 1)$; clayey silt/silt \\
\hline 86 & $13 \times-3,71-75$ & 4 & Gray $(5 Y 5 / 1)$; silt \\
\hline 87 & $14 X-1,+0-7$ & ++7 & Gray(5Y5/1); silt \\
\hline 88 & $14 \mathrm{X}-1,87-88$ & 1 & Light brownish gray (5YR6/1): clayey silt \\
\hline 89 & $14 X-1.136-147$ & $6 / 5$ & $\begin{array}{l}\text { Very dark gray(N2)/olive gray }(5 Y 4 / 1) \text {; f.sand/scoria granule (maximum } \\
\text { diameter= }(\mathrm{cm})\end{array}$ \\
\hline 90 & $15 X-1.7-8$ & 1 & Olive black $(5 Y 2 / 1)$; silty v.f.sand \\
\hline 91 & $\begin{array}{l}15 \mathrm{X}-1,12-17 * \\
15 \mathrm{X}-\mathrm{CC}, * 0-5\end{array}$ & 10 & Olive black $(5 \mathrm{Y} 2 / 1)$; m.sand \\
\hline 92 & $15 \mathrm{X}-\mathrm{CC}, 10-22+$ & $12+$ & Olive black $(5 \mathrm{Y} 2 / 1)$; $\mathrm{m}$.sand \\
\hline 93 & $16 \mathrm{X}-1,+0-28$ & $++7 / 3 / 16 / 2$ & $\begin{array}{l}\text { Gray }(5 Y 5 / 1) / \text { very dark gray }(5 Y 3 / 1) / g r a y(5 Y 5 / 1) / \text { very dark gray }(5 Y 3 / 1) \text {; } \\
\text { silt/silt/silt/v.f.sand }\end{array}$ \\
\hline 94 & $16 \times-1.99-104$ & 5 & Olive gray $(5 Y 4 / 1) ;$ silt \\
\hline 95 & $16 \times-1.106$ & $<1$ & Olive gray $(5 \mathrm{Y} 4 / 1) ;$ silt( $(5 \mathrm{~mm}$ thick $)$ \\
\hline 96 & $16 \times-2,8-12$ & 4 & Olive gray $(5 Y 5 / 2)$; silt \\
\hline 97 & $16 X-2,35-38$ & 3 & Gray(5Y5/1); silt \\
\hline 98 & $16 \times-4.63-67$ & $1 / 3$ & Dark olive gray $(5 Y 3 / 2)$ /olive gray $(5 Y 4 / 1)$; silt \\
\hline 99 & $16 X-5.52-66$ & 14 & Very dark gray $(5 \mathrm{Y} 3 / 1): \mathrm{m}$.sand \\
\hline 100 & $16 \times-5.118-120$ & 2 & Light olive gray $(5 \mathrm{Y} 6 / 1)$; silt \\
\hline 101 & $16 \times-6,17-20$ & 3 & Olive gray $(5 \mathrm{Y} 4 / 2)$; silt \\
\hline 102 & I $6 \mathrm{X}-\mathrm{CC}, 0-4$ & 4 & Dark olive gray $(5 \mathrm{Y} 3 / 2)$; silt \\
\hline 103 & $16 \mathrm{X}-\mathrm{CC}, 18-20$ & $1 / 1$ & Gray $(5 Y 5 / 1) / d a r k$ olive gray $(5 Y 3 / 2)$; silt \\
\hline 104 & $17 X-1,130-145$ & 15 & Olive gray $(5 Y 4 / 1)$; silt-sandy silt(grading) \\
\hline 105 & $17 \times-7,27-32$ & $2 / 2 / 1$ & Very dark gray $(5 Y 3 / 1) /$ olive gray $(5 Y 4 / 2) /$ olive black $(5 Y 2 / 1)$; silt/silt/m.sand \\
\hline 106 & $17 \mathrm{X}-\mathrm{CC}, 34-35$ & 1 & Light olive gray $(5 \mathrm{Y} 6 / 1)$; silt \\
\hline 107 & $18 \times-1,145$ & $<1$ & Silt \\
\hline 108 & $18 X-3135-136$ & 1 & Silt \\
\hline 109 & $18 \times-4,58-60$ & 2 & Very light gray $(\mathrm{N} 8)$; silt \\
\hline 110 & $18 \times-4,100$ & $<1$ & Very light gray(N8); silt \\
\hline iii & $18 \times-4,123-124$ & 1 & Very light gray(N8): silt \\
\hline 112 & $19 \mathrm{X}-1,46-53$ & 7 & Olive gray $(5 Y 4 / 2)$ silt \\
\hline 113 & $19 X-1.65-68$ & 3 & Olive gray $(5 Y 4 / 2)$ silt \\
\hline 114 & $19 \times-1,94-96$ & 2 & Gray $(5 Y 5 / 1)$; silt \\
\hline 115 & $19 \times-2,98-106$ & 8 & Gray $(5 Y 5 / 1)$; silt \\
\hline 116 & $19 X-3,52-60$ & 8 & Olive gray $(5 \mathrm{Y} 4 / 2)$; clayey silt \\
\hline 117 & $19 X-3,81-85$ & & Olive gray $(5 Y 4 / 2)$; clayey silt \\
\hline \multirow[t]{2}{*}{118} & $19 \times-4,92-150^{*}$ & $36 / 14 / 11$ & $\begin{array}{l}\text { Dark gray(N3)/grayish black(N2)/olive black(5Y2/1); pebble-granule gravel } \\
\text { with sandy silt }\end{array}$ \\
\hline & $19 X-5, * 0-3$ & & \\
\hline & $19 X-5,57-59$ & 2 & Olive gray $(5 Y 4 / 2)$; silt \\
\hline 120 & $19 \times-6,13-14$ & i & Medium fight gray(N6); silt \\
\hline 121 & $19 X-6,20$ & ${ }_{4}^{1}$ & Medium light gray(N6); silt pocket \\
\hline 122 & $19 \times-6,39-45$ & $4 / 2$ & Olive gray $(5 Y 5 / 2)$ /light olive gray $(5 Y 6 / 1) ;$ silt \\
\hline 123 & $20 X-2,63-150^{*}$ & 217 & Pumice gravel scattered in sandy mud (maximum diameter $=4 \mathrm{~cm}$ ) \\
\hline 124 & $20 \times-C C, 28-50+$ & $6 / 16+$ & $\begin{array}{l}\text { Light olive gray }(5 Y 6 / 1) / g r a y i s h \text { black(N2); sand/scoria (maximum } \\
\text { diameter }=2 \mathrm{~cm} \text { ) }\end{array}$ \\
\hline
\end{tabular}

Note: Sample 126-790B-10H-6, $140 \mathrm{~cm}$, is correlative to $126-790 \mathrm{C}-1 \mathrm{H}-4.57 \mathrm{~cm}$. v.f. $=$ very fine, v.f.s. $=$ very fine sand, m.s. $=$ medium sand, f. $=$ fine, $m$. $=$ medium, and $\mathrm{c}=$ coarse. The list for forearc Site 792 is given in Fujioka et al. (this volume).

${ }^{*}$ Continue to the next * below.

+ Continue to the next + or ++ below, 
Table 2. Petrographic characteristics of backarc tephras at Sites 790/791.

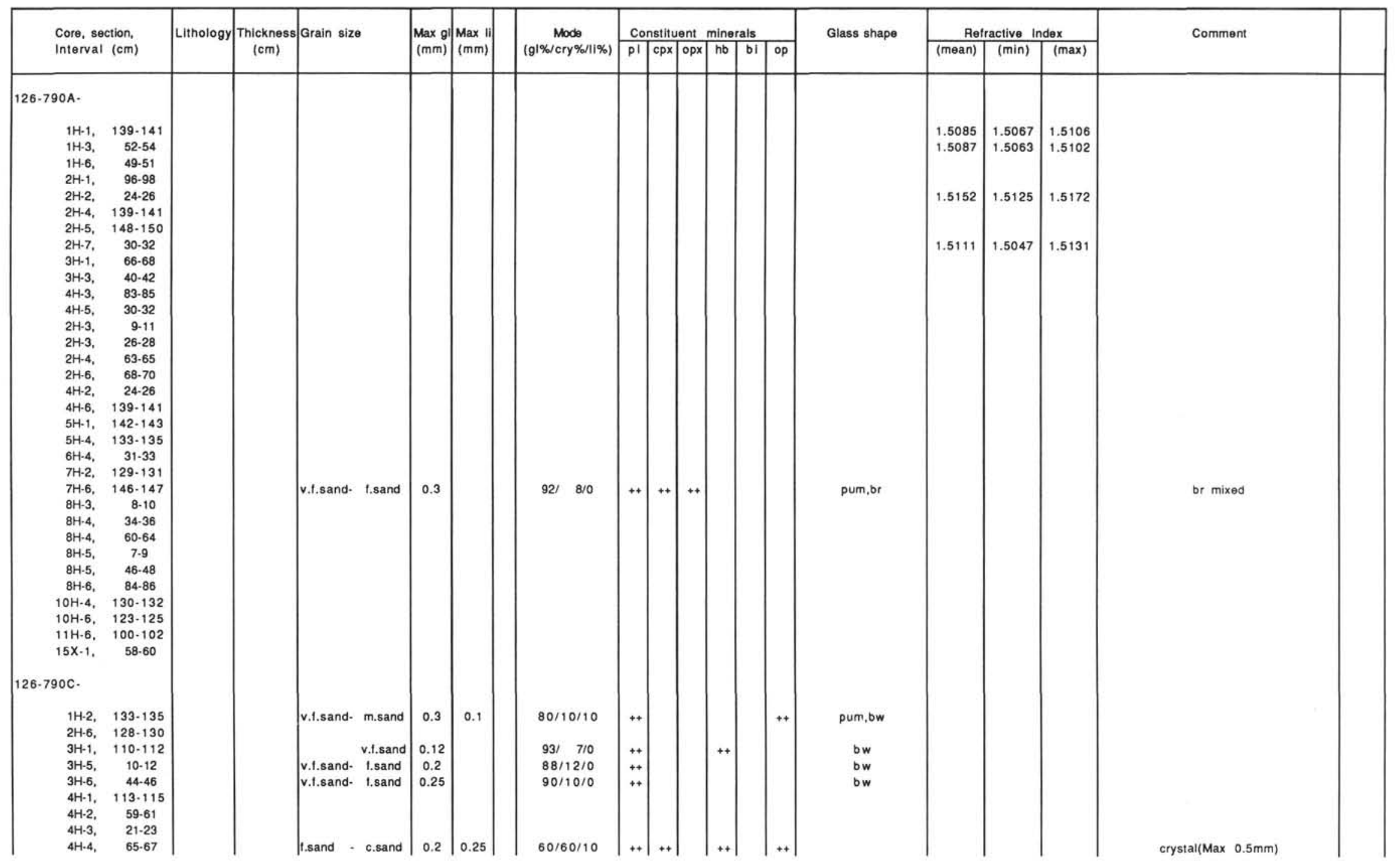


Table 2 (continued).

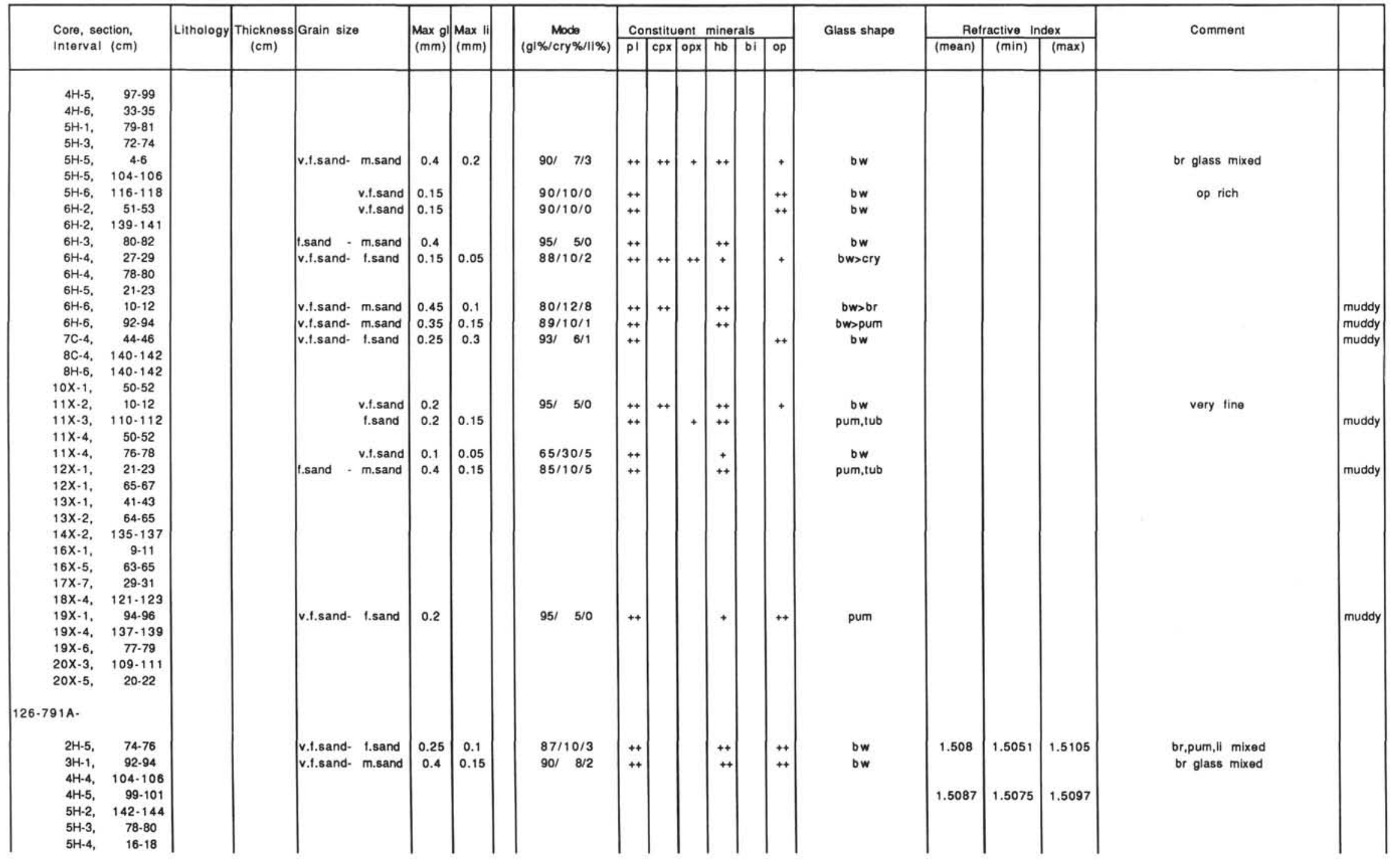


Table 2 (continued).

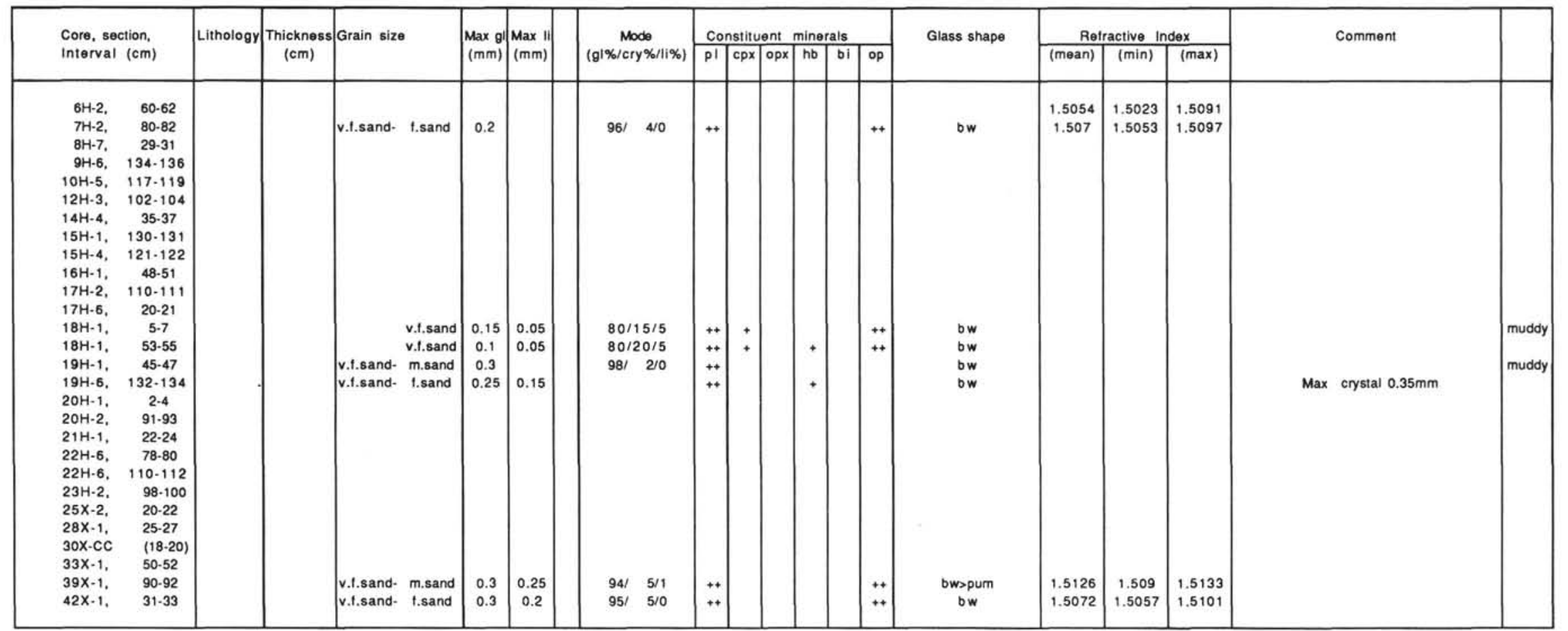


A

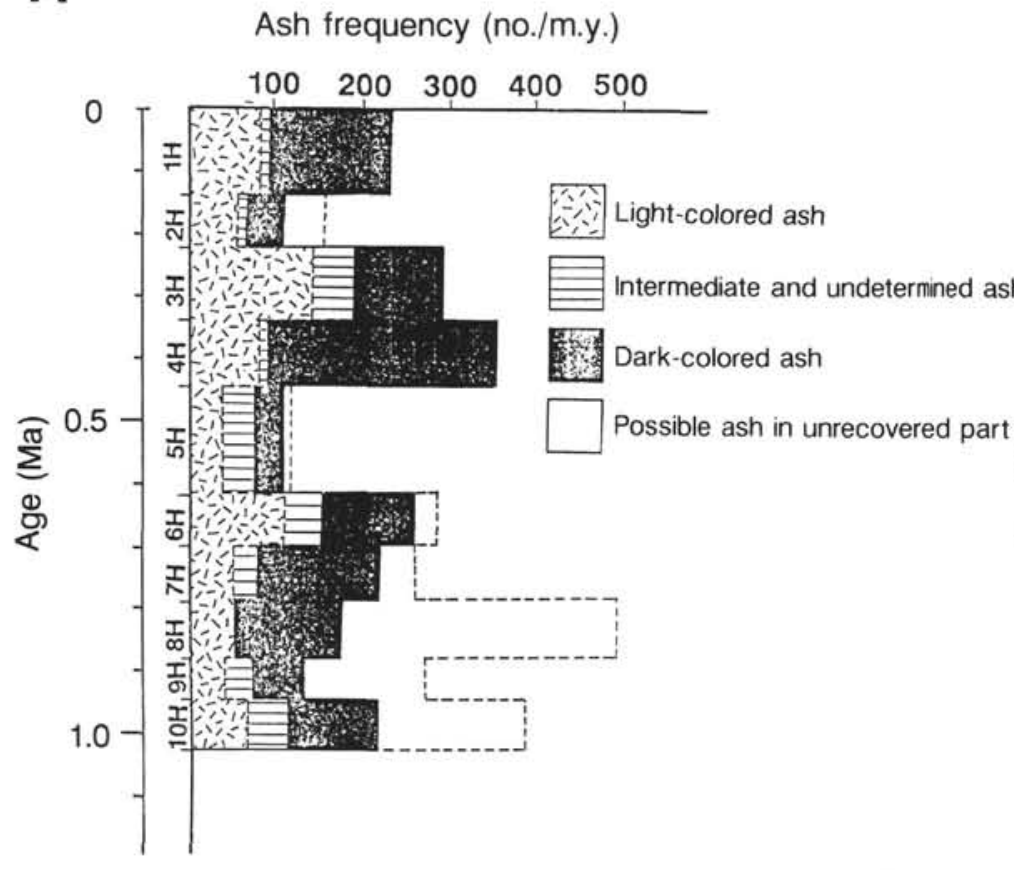

B

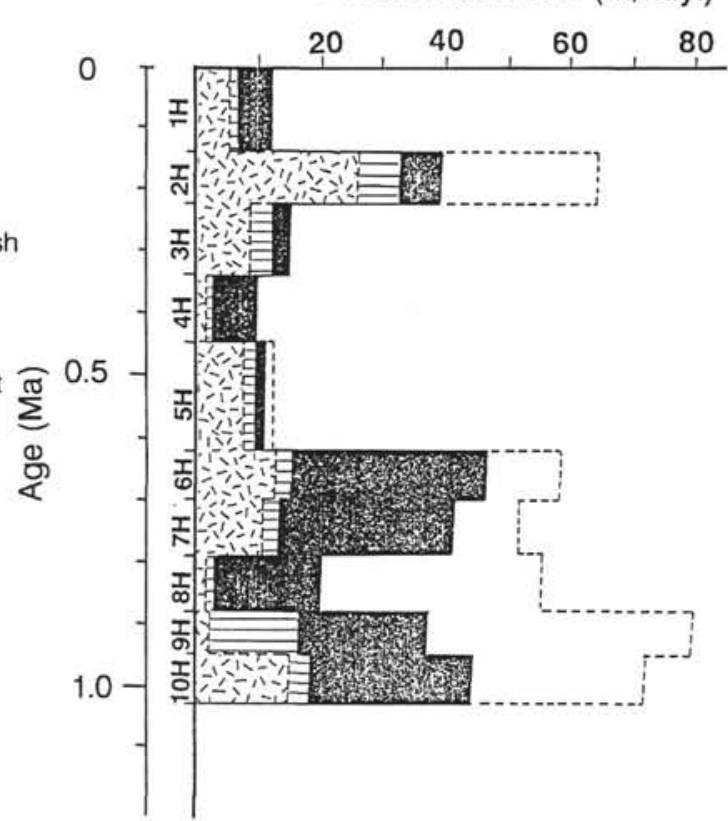

Figure 5. Accumulation rate of the Quaternary volcaniclastic materials of forearc Site 792. A. Ash frequency indicated by ash number per million years, calculated by the constant sedimentation rates of muddy hemipelagites. B. Ash sedimentation rate obtained by the same method as in A.

A

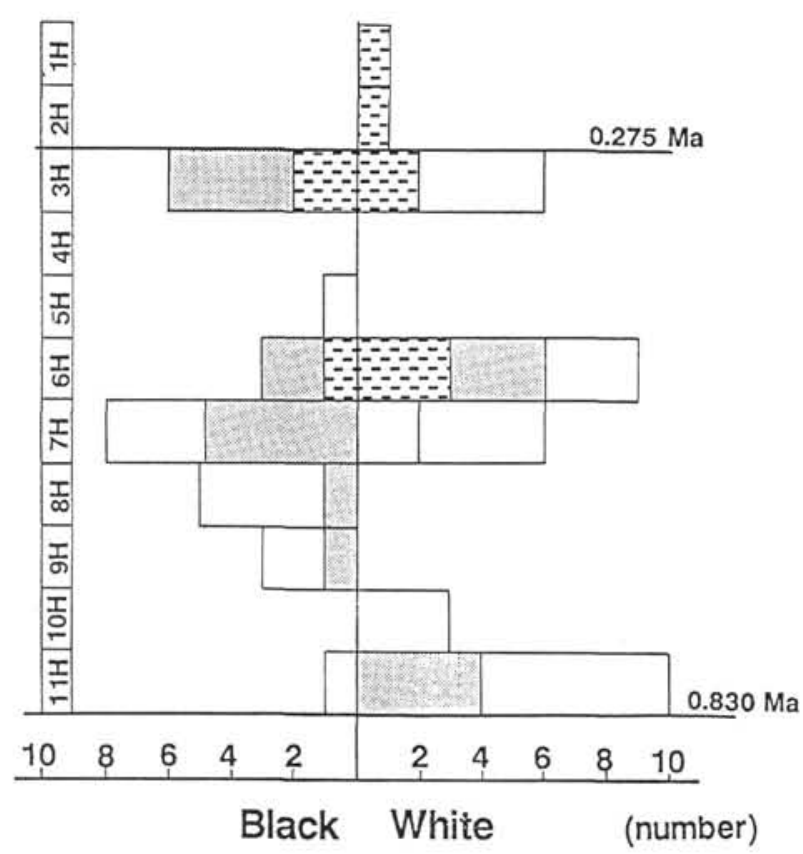

B

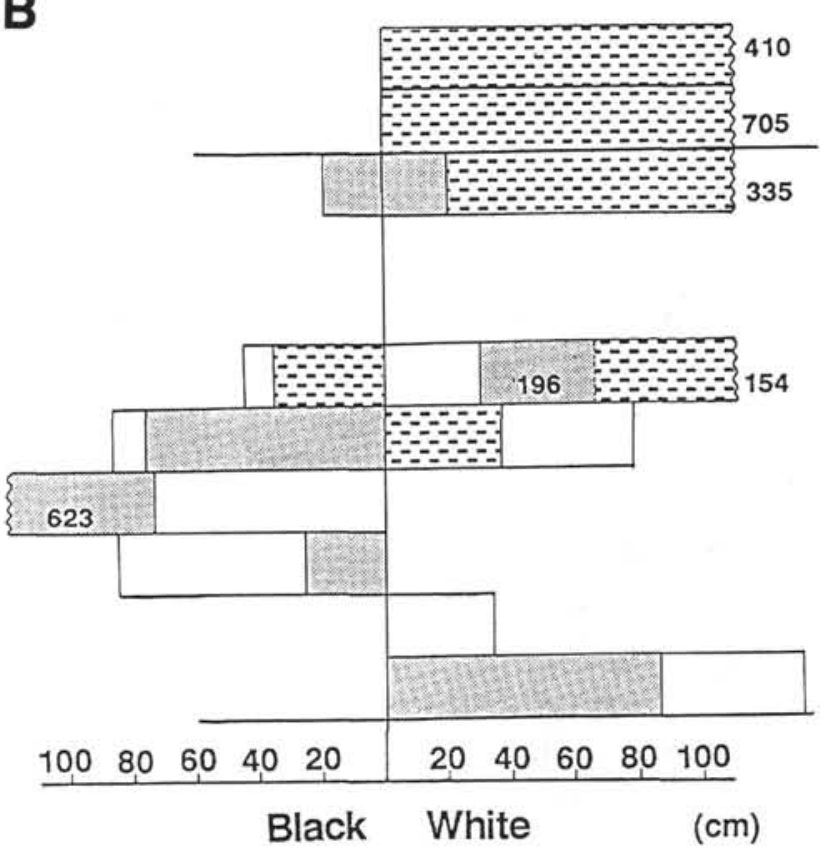
Granule size Sand size

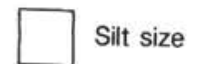

Figure 6. Frequency (A) and thickness (B) diagram of the Quaternary tephras at forearc Site 793. 
A

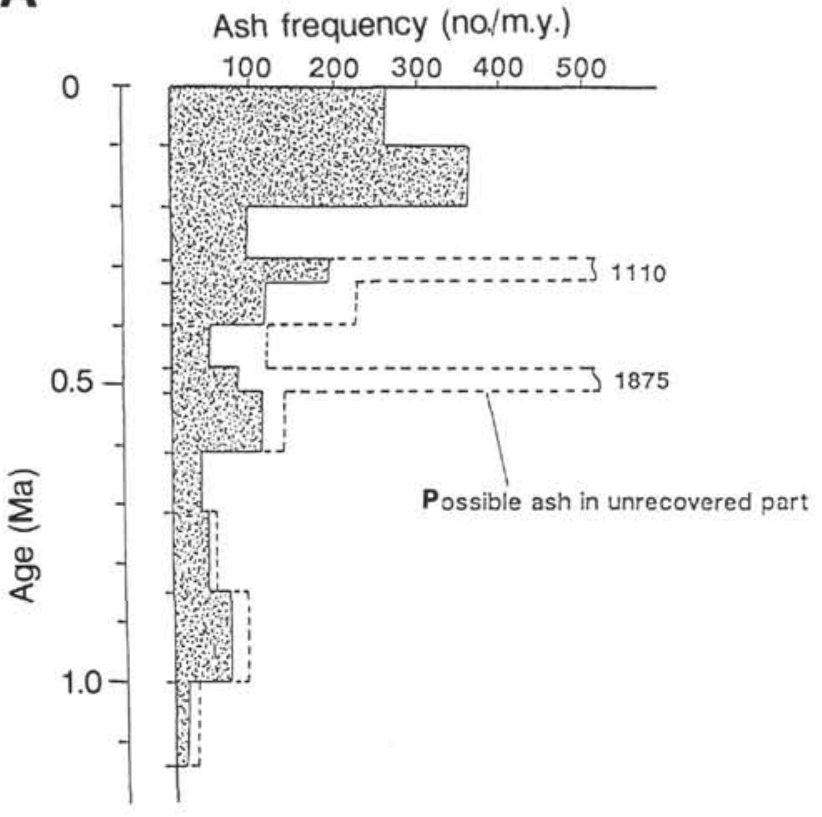

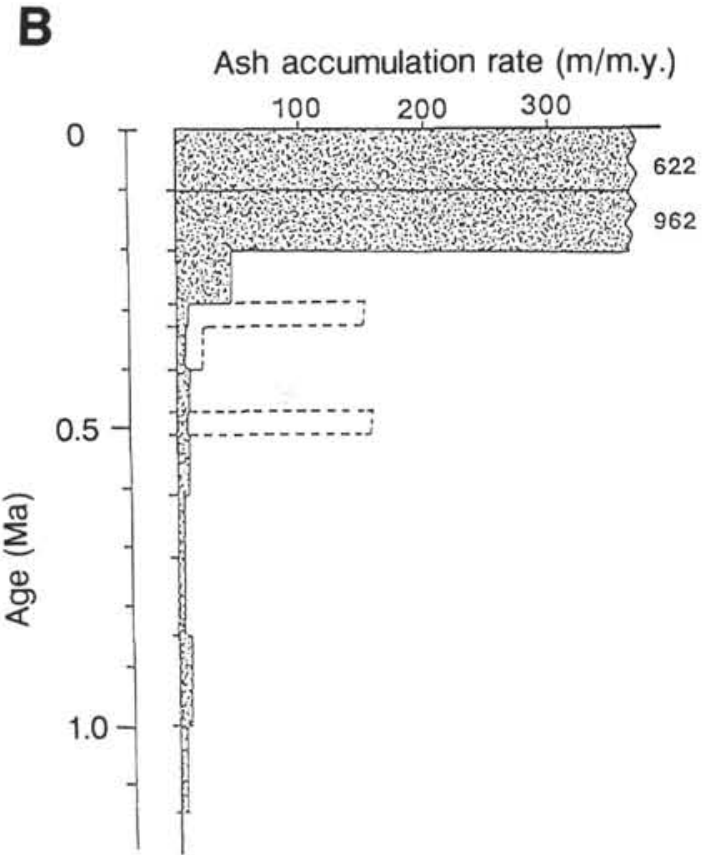

Figure 7. Frequency (A) and accumulation (B) diagram of the tephras at backarc Sites 790/791.

diameters of the pumice fragments range from 200 to $600 \mu \mathrm{m}$. Examples of this type include Samples 126-791A-22H-6, 28-32 cm, and 126-791A-4H-3, 6-10 cm (Fig. 9). This type of grain-size distribution represents resedimented deposits (Sparks et al., 1981; Taylor, Fujioka, et al., 1990). In contrast, the fine glassy tephras have a wellsorted distribution curve, with median diameters ranging from 60 to $100 \mu \mathrm{m}$. Examples of this type include Samples 126-790B-7H-6, $146-147 \mathrm{~cm}$, and 126-790C-7H-4, 44-46 cm (Fig. 9). Fine glassy ashes are often associated with coarse thick pumiceous tephras, suggesting they originate from a subaqueous eruption (Fisher, 1984; Kokelaar et al., 1985; Fiske, 1986; Fiske and Cashman, 1989).

\section{CHEMISTRY OF THE VOLCANIC GLASS SHARDS}

Tephras from Leg 126 sites belong to the low-alkali tholeiitic series on Kuno's silica-alkali diagram (Figs. 10-12; Kuno, 1966). Generally, tephras at Site 788 are slightly different from those at the backarc and forearc sites in several respects. First, the tephras at Site 788 have very low $\mathrm{SiO}_{2}, \mathrm{Na}_{2} \mathrm{O}$, and $\mathrm{K}_{2} \mathrm{O}$ concentrations (Table 4). Second, at the forearc and backarc sites, tephras extremely enriched in $\mathrm{K}_{2} \mathrm{O}$ were recognized in some intervals. Sometimes $\mathrm{K}_{2} \mathrm{O}$ contents exceeded $4 \mathrm{wt} \%$. These kinds of tephras may possibly come from the western regions, as discussed below.

The total alkali content of Site 788 tephras is quite constant, that is, $\mathrm{Na}_{2} \mathrm{O}$ and $\mathrm{K}_{2} \mathrm{O}$ change sympathetically with time. The total alkali contents of Site 790 tephras vary only slightly, but the youngest ones are quite low in $\mathrm{K}_{2} \mathrm{O}, \mathrm{Na}_{2} \mathrm{O}$, and total alkali. Tephras at Sites 792 and 793 , however, vary greatly over time. See Appendix (on microfiche in back pocket) for the chemical composition data of the volcanic glasses at arc Site 788 and backarc Sites 790/791.

\section{CORRELATION OF THE ASH LAYERS}

Establishing a correlation of the volcanic ash layers is necessary to determine the volcanic history in the Izu-Bonin areas as well as in other regions around the Japanese Islands. Although most of the 300 tephras identified in this study have different but similar chemical and mineralogical compositions, several have quite distinct characteris- tics that are comparable to tephras identified in other studies. Here we show four examples of correlations of tephras from the Izu-Bonin forearc to the other sites and volcanic islands. The distinctive properties of these volcanic ash layers are listed in Table 5 .

\section{Samples 126-792A-3H-1, 80-94 cm, and 126-793A-4H-2, 96-98 cm}

These tephras have similar refractive indices and chemical compositions as well as glass shard types and mineral assemblages. Their age is estimated to be equivalent to Zone CN14b.

\section{Samples 126-792A-5H-3, 131-133 cm, and $442 \mathrm{~A}-5-3,30-34 \mathrm{~cm}$}

These two ash layers have similar refractive indices and chemical compositions as well as similar glass shard types and mineral assemblages. Therefore, these two ashes may be correlative with each other although they are several hundred kilometers apart. The origin of this tephra may be further west of the Shikoku Basin, for example, from the Ryukyu Arc.

\section{Black Tephras at Site 792 and Aogashima Volcanics}

The chemical compositions of the Kurosaki volcanics, in the lowermost sequence of Aogashima Island, have distinctly different chemical characteristics as compared with younger sequences, that is, low $\mathrm{TiO}_{2}$ and $\mathrm{K}_{2} \mathrm{O}$ contents and high $\mathrm{FeO}$ and $\mathrm{CaO}$ contents (Takada et al., in press). The chemical compositions of some black volcanic ash layers at Site 792 are quite similar to those of the Kurosaki volcanics. Examples include Samples 126-792A-4H-1, 117-119 cm, and $126-792 \mathrm{~A}-4 \mathrm{H}-3,20-22 \mathrm{~cm}$.

\section{Samples 126-792A-1H-5, 81-83 cm, and Aso-4}

The white coarse volcanic ash layer at Hole 792A has quite similar mineral assemblages, refractive indices, and chemical compositions as well as ages and glass shard shapes to that of Aso-4 (Furuta et al., 1986) of the Aso Volcano, Kyushu. The type of glass shard in the 

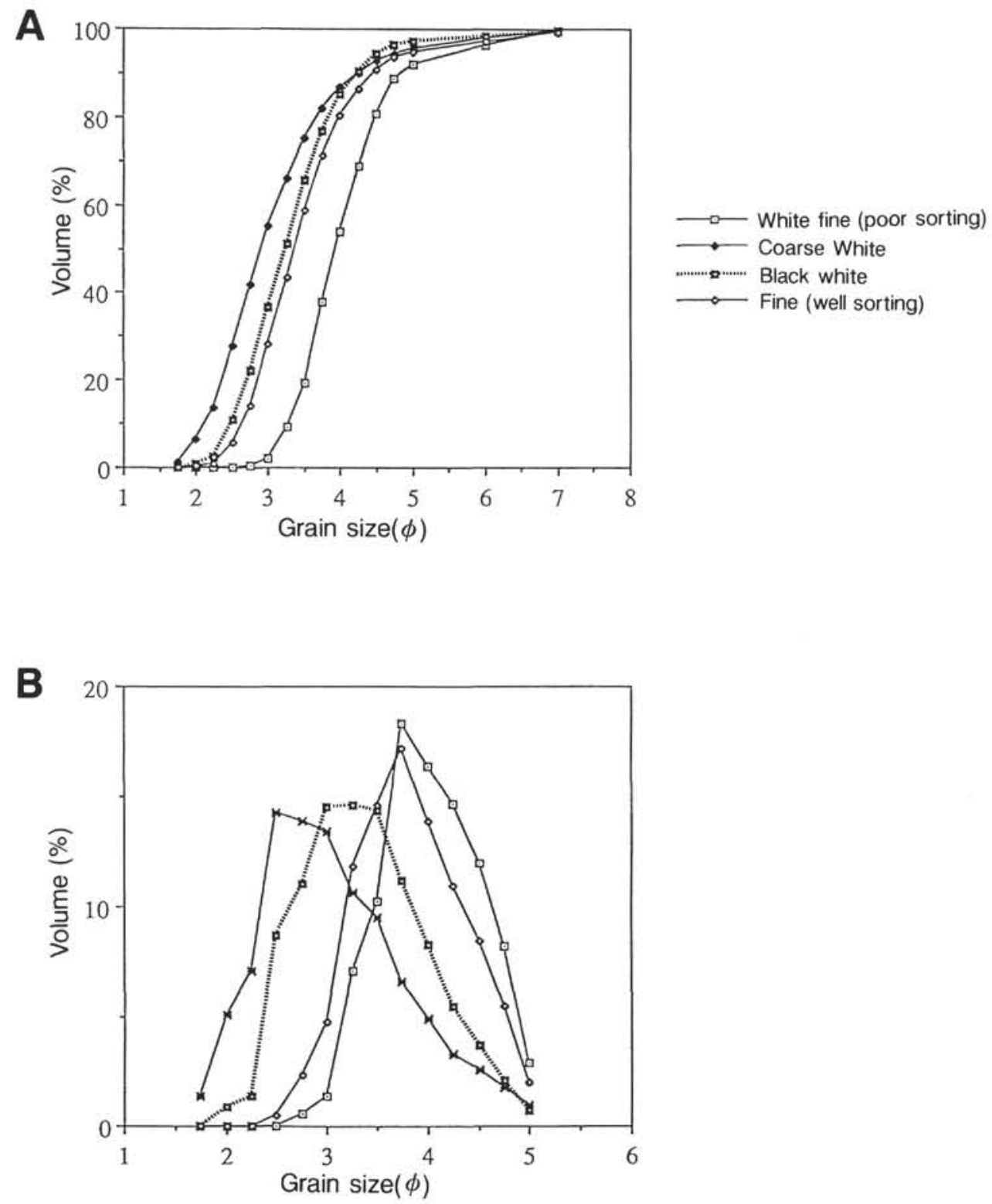

Figure 8. Grain-size characteristics of four types of tephras. A. Cumulative frequency grain-size curve for all the tephras at Site 792, Izu-Bonin forearc. B. Frequency grain-size distribution curve for volcanic ash layers at Site 792. Scale of the grain size is represented by $\phi(\mathrm{phi})$.

Aso- 4 is bubble wall with a pale brown color. The age of this tephra is estimated to be $70-80 \mathrm{ka}$ according to stratigraphic data obtained on land (Machida and Arai, 1983) and around $80 \mathrm{ka}$ according to U-Th age determinations (Omura, 1984).

\section{DISCUSSION}

The chemical characteristics of the tephras from the PliocenePleistocene ages have low $\mathrm{K}_{2} \mathrm{O}$ contents similar to the keratophyre of the Paleozoic rift as well as low-K rhyolite in the rift area (Gill, 1981; Gill et al., 1984; Reagan and Meijer, 1984; Hawkins and Melchior, 1985; Brouxel et al., 1987; Hochstaedter et al., 1990a, 1990b).

Volcanism in this stage shows the characteristic basaltic and rhyolitic bimodal distribution in both chemical and visual compositions. This kind of bimodal volcanism was noted in the basin and range province of the Cascade region of the North America, where extensional stress fields predominated from Eocene to Holocene times (Christiansen and Lipman, 1972; Eaton, 1982, 1984). Kennett et al. (1979) noted the characteristic volcanic pulse of the Quaternary period in the circum-Pacific regions and named this pulse "Cascadian." The Izu-Bonin explosive volcanism is also similar in character to the Cascadian event.

The numbers of Pliocene-Pleistocene volcanic ash layers increased in this area, and thick tephra piles accumulated (Fujioka et al., this volume). From the grain-size distribution and chemical compositions, we determined that the tephras came mostly from along the present IzuBonin Arc, although some tephras possibly came from a more western part of the Philippine Sea (i.e., from the Ryukyu Arc).

Along the Ryukyu Arc, the late Miocene to Pliocene Shimajiri Group yielded many coarse tephras including pumices (Konishi, 


\begin{tabular}{|c|c|c|c|c|c|c|c|c|c|c|c|c|c|c|}
\hline Site & & $788 \mathrm{C}$ & $788 \mathrm{C}$ & & $790 \mathrm{~A}$ & $790 \mathrm{~A}$ & $790 \mathrm{~A}$ & $790 \mathrm{~A}$ & $790 \mathrm{~A}$ & $790 \mathrm{~A}$ & $790 \mathrm{~A}$ & & $790 B$ & $790 \mathrm{~B}$ \\
\hline Core, section & & $11 \mathrm{H}-3$ & $11 \mathrm{H}-4$ & & $1 \mathrm{H}-1$ & $1 \mathrm{H}-3$ & $2 \mathrm{H}-1$ & $2 \mathrm{H}-2$ & $2 \mathrm{H}-4$ & $2 \mathrm{H}-5$ & $3 \mathrm{H}-1$ & & $2 \mathrm{H}-3$ & $2 \mathrm{H}-3$ \\
\hline Interval $(\mathrm{cm})$ & & $38-40$ & $108-110$ & & $139-141$ & $52-54$ & $96-98$ & $24-26$ & $139-141$ & $148-150$ & $66-68$ & & 11-Sep & $26-28$ \\
\hline \multirow[t]{17}{*}{$\begin{array}{c}\text { Cumulative } \\
\text { volume percentages }\end{array}$} & $\begin{array}{c}\text { Class interval } \\
(\phi)\end{array}$ & & & & & & & & & & & & & \\
\hline & 1.75 & 0 & 0 & & 0 & 0 & 0 & 0 & 0 & 0 & 2 & & 0 & 0 \\
\hline & $2.00-1.75$ & 0 & 1.2 & & 0 & 0 & 0 & 0 & 0.7 & 0 & 8.4 & & 0 & 0 \\
\hline & $2.25-2.00$ & 0.1 & 3.1 & & 0 & 0 & 0 & 0 & 1.8 & 0 & 17.3 & & 0 & 0 \\
\hline & $2.50-2.25$ & 3.3 & 14 & & 0 & 1.3 & 0.5 & 1.8 & 9.4 & 0.2 & 17.3 & & 0 & 0 \\
\hline & $2.75-2.50$ & 11.1 & 26.9 & & 0.5 & 5.3 & 3.1 & 6.9 & 20.5 & 1.8 & 31.7 & & 0.9 & 0.8 \\
\hline & $3.00-2.75$ & 25.5 & 42.8 & & 1.8 & 13.3 & 8.8 & 16.8 & 36.7 & 5.4 & 45.1 & & 3.1 & 2.8 \\
\hline & $3.25-3.00$ & 41.8 & 57.2 & & 8.6 & 27 & 24.1 & 30.9 & 52.8 & 17.2 & 57.3 & & 12.9 & 11.5 \\
\hline & $3.50-3.25$ & 58.5 & 70.7 & & 18.3 & 42.7 & 42.8 & 46.3 & 68.4 & 32.1 & 66.6 & & 26.1 & 23.1 \\
\hline & $3.75-3.50$ & 71.7 & 79.7 & & 35.2 & 58.7 & 62.6 & 60.8 & 79 & 49.2 & 74.9 & & 45.1 & 39.2 \\
\hline & $4.00-3.75$ & 81.3 & 86 & & 50.6 & 71.2 & 77.5 & 72.1 & 86.4 & 63.1 & 80.9 & & 61.3 & 53 \\
\hline & $4.25-4.00$ & 87.6 & 89.7 & & 64.6 & 80.5 & 87.8 & 80.4 & 90.9 & 73.9 & 85.6 & & 74.9 & 64.7 \\
\hline & $4.50-4.25$ & 92 & 92.3 & & 76.8 & 87.5 & 94.3 & 86.8 & 94 & 82.2 & 89.1 & & 85.3 & 74.3 \\
\hline & $4.75-4.50$ & 94.7 & 93.9 & & 85.7 & 92.1 & 97.7 & 91.1 & 95.8 & 87.7 & 91.9 & & 92.1 & 81.1 \\
\hline & $5.00-4.75$ & 95.6 & 94.6 & & 89.7 & 93.8 & 98.2 & 92.8 & 96.4 & 89.6 & 92.7 & & 94.3 & 84 \\
\hline & $5.00-6.00$ & 97.3 & 97 & & 95.6 & 96.6 & 99.1 & 96.2 & 98 & 93.6 & 95 & & 97.7 & 91.2 \\
\hline & $7.00-6.00$ & 99.3 & 99.1 & & 99.4 & 99.4 & 99.8 & 99.3 & 99.6 & 98.2 & 97.2 & & 99.8 & 97.7 \\
\hline Site & & $790 \mathrm{~B}$ & $790 \mathrm{~B}$ & $790 B$ & $790 B$ & $790 \mathrm{~B}$ & $790 \mathrm{~B}$ & $790 B$ & $790 B$ & $790 B$ & $790 B$ & $790 \mathrm{~B}$ & $790 B$ & \\
\hline Core, section & & $2 \mathrm{H}-4$ & $2 \mathrm{H}-6$ & $4 \mathrm{H}-2$ & $4 \mathrm{H}-6$ & $7 \mathrm{H}-2$ & $7 \mathrm{H}-6$ & $8 \mathrm{H}-3$ & $8 \mathrm{H}-4$ & $8 \mathrm{H}-4$ & $8 \mathrm{H}-5$ & $8 \mathrm{H}-5$ & $8 \mathrm{H}-6$ & \\
\hline Interval (cm) & & $63-65$ & $68-70$ & $24-26$ & $139-141$ & $129-131$ & $146-148$ & 10-Aug & $34-36$ & $60-62$ & 7- 9 & $46-48$ & $84-86$ & \\
\hline \multirow[t]{17}{*}{$\begin{array}{c}\text { Cumulative } \\
\text { volume percentages }\end{array}$} & $\begin{array}{c}\text { Class interval } \\
(\phi)\end{array}$ & & & & & & & & & & & & & \\
\hline & 1.75 & 0 & 0 & 0 & 0 & 0 & 0 & 0 & 0 & 0 & 0.7 & 0 & 0 & \\
\hline & $2.00-1.75$ & 0 & 0 & 0 & 1.1 & 0 & 0 & 0 & 0 & 0 & 5 & 2.1 & 0 & \\
\hline & $2.25-2.00$ & 0 & 0 & 0 & 2.8 & 0 & 0 & 0 & 0 & 0 & 11.3 & 5.3 & 0 & \\
\hline & $2.50-2.25$ & 0 & 1.4 & 2 & 12.1 & 1.5 & 1.9 & 1.3 & 1.9 & 0.5 & 32.3 & 21.8 & 0 & \\
\hline & $2.75-2.50$ & 0.4 & 5.7 & 7.5 & 22.9 & 5.9 & 7.4 & 5.1 & 7.2 & 3 & 52.2 & 38.7 & 0.3 & \\
\hline & $3.00-2.75$ & 1.5 & 14.2 & 18.1 & 36.1 & 14.5 & 18.2 & 12.7 & 17.5 & 8.4 & 70.6 & 56.4 & 1 & \\
\hline & $3.25-3.00$ & 7.4 & 29 & 32.8 & 49.3 & 28.4 & 34.5 & 25.9 & 32.3 & 23.2 & 81.7 & 70.1 & 6.1 & \\
\hline & $3.50-3.25$ & 15.9 & 45.9 & 48.9 & 62.3 & 44.2 & 52.4 & 41.3 & 48.7 & 41.4 & 90 & 82 & 14.3 & \\
\hline & $3.75-3.50$ & 32 & 62.6 & 63.8 & 72.9 & 59.9 & 67.7 & 58.2 & 64.2 & 60.8 & 93.2 & 88.8 & 33.9 & \\
\hline & $4.00-3.75$ & 47 & 75.3 & 75.3 & 80.9 & 72.3 & 78.9 & 71.7 & 76 & 75.4 & 95.3 & 93.3 & 52 & \\
\hline & $4.25-4.00$ & 61.1 & 84.3 & 83.7 & 86.6 & 81.5 & 86.1 & 82.1 & 84.4 & 85.6 & 96.3 & 95.7 & 68.6 & \\
\hline & $4.50-4.25$ & 73.8 & 90.6 & 89.9 & 91 & 88.4 & 91.1 & 89.5 & 90.3 & 92.2 & 97.1 & 97.3 & 81.1 & \\
\hline & $4.75-4.50$ & 83.3 & 94.3 & 93.9 & 93.9 & 92.8 & 94 & 94 & 93.8 & 95.8 & 97.6 & 98.1 & 89.1 & \\
\hline & $5.00-4.75$ & 87.5 & 95.4 & 95.2 & 94.9 & 94.2 & 94.9 & 95.2 & 94.9 & 96.6 & 97.8 & 98.3 & 91.3 & \\
\hline & $5.00-6.00$ & 94.6 & 97.3 & 97.3 & 97.2 & 96.6 & 96.7 & 97.2 & 96.9 & 97.9 & 98.5 & 98.9 & 94.7 & \\
\hline & $7.00-6.00$ & 99.3 & 99.6 & 99.4 & 99.3 & 99 & 99 & 99.6 & 99.2 & 99.7 & 99.1 & 99.5 & 99.3 & \\
\hline
\end{tabular}


Table 3 (continued).

\begin{tabular}{|c|c|c|c|c|c|c|c|c|c|c|c|c|c|}
\hline Site & $790 \mathrm{C}$ & $790 \mathrm{C}$ & $790 \mathrm{C}$ & $790 \mathrm{C}$ & $790 \mathrm{C}$ & $790 \mathrm{C}$ & $790 \mathrm{C}$ & $790 \mathrm{C}$ & $790 \mathrm{C}$ & $790 \mathrm{C}$ & $790 \mathrm{C}$ & $790 \mathrm{C}$ & $790 \mathrm{C}$ \\
\hline Core, section & $3 \mathrm{H}-5$ & $3 \mathrm{H}-6$ & $5 \mathrm{H}-3$ & $5 \mathrm{H}-5$ & $5 \mathrm{H}-6$ & $6 \mathrm{H}-2$ & $6 \mathrm{H}-2$ & $6 \mathrm{H}-3$ & $6 \mathrm{H}-4$ & $6 \mathrm{H}-4$ & $6 \mathrm{H}-6$ & $6 \mathrm{H}-6$ & $7 \mathrm{H}-2$ \\
\hline Interval $(\mathrm{cm})$ & $12-$ Oct & $44-46$ & $72-74$ & 6-Apr & $116-118$ & $51-53$ & $139-141$ & $80-82$ & $27-29$ & $78-80$ & $12-\mathrm{Oct}$ & $92-94$ & $44-46$ \\
\hline
\end{tabular}

\begin{tabular}{|c|c|c|c|c|c|c|c|c|c|c|c|c|c|c|}
\hline \multirow[t]{17}{*}{$\begin{array}{c}\text { Cumulative } \\
\text { volume percentages }\end{array}$} & $\begin{array}{c}\text { Class interval } \\
(\phi)\end{array}$ & & & & & & & & & & & & & \\
\hline & $1.75-$ & 0 & 0 & 0 & 0 & 0 & 0 & 0 & 0 & 0 & 0 & 0 & 0 & 0 \\
\hline & $2.00-1.75$ & 0 & 0 & 0 & 0.3 & 0 & 0 & 1.6 & 0 & 0 & 1.5 & 0 & 0 & 0 \\
\hline & $2.25-2.00$ & 0 & 0 & 0 & 0.8 & 0 & 0 & 4.1 & 0 & 0 & 3.9 & 0 & 0 & 0 \\
\hline & $2.50-2.25$ & 0 & 0 & 0.9 & 5.9 & 0 & 0 & 17.4 & 0.1 & 0 & 17 & 0 & 0.5 & 0 \\
\hline & $2.75-2.50$ & 0.6 & 0.6 & 3.9 & 14.8 & 0.7 & 0.8 & 31.6 & 1.5 & 1.1 & 31.7 & 0.9 & 2.7 & 0.7 \\
\hline & $3.00-2.75$ & 2.1 & 2.1 & 10 & 29.4 & 2.4 & 2.7 & 47.3 & 4.9 & 3.8 & 48.9 & 3.1 & 7.4 & 2.4 \\
\hline & $3.25-3.00$ & 9.6 & 9.6 & 22.5 & 45.2 & 10.8 & 11.7 & 60.6 & 16.9 & 15.3 & 63.3 & 12.9 & 20 & 10.4 \\
\hline & $3.50-3.25$ & 20.1 & 20.2 & 37.3 & 61.1 & 22.6 & 23.9 & 72.8 & 32.2 & 30.4 & 76.4 & 26.1 & 35.4 & 21.4 \\
\hline & $3.75-3.50$ & 37.8 & 38 & 52.8 & 73.4 & 42 & 42.6 & 80.9 & 50.2 & 49.9 & 84.4 & 45.2 & 52.5 & 38.3 \\
\hline & $4.00-3.75$ & 53.4 & 53.6 & 64.9 & 82.3 & 58.5 & 58.4 & 86.7 & 64.8 & 65.6 & 89.9 & 61.4 & 65.9 & 53.1 \\
\hline & $4.25-4.00$ & 67 & 67.2 & 73.9 & 88 & 72.3 & 71.5 & 90.3 & 76.2 & 77.8 & 93 & 74.8 & 75.8 & 66 \\
\hline & $4.50-4.25$ & 78.2 & 78.3 & 80.8 & 92 & 82.6 & 81.6 & 92.9 & 84.9 & 86.6 & 95.1 & 84.8 & 83.2 & 77.1 \\
\hline & $4.75-4.50$ & 86 & 86 & 85.4 & 94.3 & 89.2 & 88.3 & 94.5 & 90.6 & 92 & 96.3 & 91.1 & 87.9 & 85.2 \\
\hline & $5.00-4.75$ & 89.3 & 89.2 & 87.3 & 95.1 & 91.5 & 90.7 & 95.2 & 92.6 & 93.6 & 96.7 & 93 & 89.6 & 88.9 \\
\hline & $5.00-6.00$ & 95.1 & 95 & 92 & 97.1 & 95.5 & 95.1 & 97.1 & 96 & 96.2 & 97.8 & 96 & 93.8 & 95.4 \\
\hline & $7.00-6.00$ & 99.3 & 99.4 & 97.3 & 99.1 & 99.2 & 99.1 & 99.1 & 99.2 & 99.6 & 99.4 & 99.3 & 98.4 & 99.6 \\
\hline Site & & $790 \mathrm{C}$ & $790 \mathrm{C}$ & $790 \mathrm{C}$ & $790 \mathrm{C}$ & $790 \mathrm{C}$ & $790 \mathrm{C}$ & $790 \mathrm{C}$ & $790 \mathrm{C}$ & $790 \mathrm{C}$ & $790 \mathrm{C}$ & $790 \mathrm{C}$ & $790 \mathrm{C}$ & \\
\hline Core, section & & $11 X-2$ & $11 X-3$ & $12 X-1$ & $12 X-1$ & $13 X-1$ & $13 x-2$ & $14 X-2$ & $16 X-1$ & $17 X-7$ & $18 X-4$ & $19 X-1$ & $20 X-5$ & \\
\hline Interval (cm) & & $12-\mathrm{Oct}$ & $110-112$ & $21-23$ & $65-67$ & $41-43$ & $64-65$ & $135-138$ & 9. 11 & $29-31$ & $121-123$ & $94-96$ & $20-22$ & \\
\hline \multirow[t]{17}{*}{$\begin{array}{c}\text { Cumulative } \\
\text { volume percentages }\end{array}$} & $\begin{array}{c}\text { Class interval } \\
(\phi)\end{array}$ & & & & & & & & & & & & & \\
\hline & $1.75-$ & 0 & 0 & 0 & 0 & 0 & 0 & 2.8 & 0 & 0 & 0 & 0 & 0 & \\
\hline & $2.00-1.75$ & 0 & 0 & 0.5 & 0 & 1.4 & 0 & 10.6 & 0 & 0 & 0 & 0 & 1.7 & \\
\hline & $2.25-2.00$ & 0 & 0 & 1.3 & 0 & 3.5 & 0 & 21.3 & 0 & 0 & 0 & 0 & 4.4 & \\
\hline & $2.50-2.25$ & 0 & 1 & 7.7 & 0 & 15.3 & 1.2 & 32.8 & 0 & 1.1 & 0 & 0 & 18.2 & \\
\hline & $2.75-2.50$ & 1.3 & 4.5 & 17.6 & 1 & 29 & 5 & 43.8 & 0 & 4.6 & 0 & 1.4 & 32.5 & \\
\hline & $3.00-2.75$ & 4.5 & 11.7 & 32.8 & 3.5 & 45.4 & 12.6 & 54.1 & 0 & 11.8 & 0 & 4.8 & 47.8 & \\
\hline & $3.25-3.00$ & 17.5 & 26.5 & 48.8 & 14.4 & 60.2 & 26.5 & 64.3 & 0 & 26 & 0 & 18.6 & 61 & \\
\hline & $3.50-3.25$ & 34.2 & 43.9 & 64.7 & 28.9 & 74.1 & 42.7 & 74.5 & 0 & 42.7 & 0 & 36.2 & 73.3 & \\
\hline & $3.75-3.50$ & 54.4 & 61.9 & 76.5 & 49 & 83.5 & 59.9 & 83.2 & 0 & 60.3 & 0 & 56.5 & 82.1 & \\
\hline & $4.00-3.75$ & 70.3 & 75.5 & 85 & 65.9 & 90 & 73.3 & 89.6 & 0 & 73.8 & 0 & 72.3 & 88.4 & \\
\hline & $4.25-4.00$ & 82.1 & 85.1 & 90.3 & 79.9 & 93.8 & 83 & 93.8 & 0 & 83.5 & 0 & 83.8 & 92.3 & \\
\hline & $4.50-4.25$ & 90.2 & 91.5 & 93.9 & 89.8 & 96.2 & 89.9 & 96.6 & 0 & 90.2 & 0 & 91.5 & 95 & \\
\hline & $4.75-4.50$ & 94.9 & 95.1 & 96 & 95.7 & 97.5 & 94 & 98.1 & 0 & 94.1 & 0 & 95.8 & 96.5 & \\
\hline & $5.00-4.75$ & 96.1 & 96.1 & 96.6 & 97.1 & 97.9 & 95.2 & 98.6 & 0 & 95.2 & 0 & 96.8 & 97.1 & \\
\hline & $5.00-6.00$ & 97.8 & 97.9 & 97.9 & 98.6 & 98.8 & 97.2 & 99.3 & 0 & 97.3 & 0 & 98.3 & 98.4 & \\
\hline & $7.00-6.00$ & 99.7 & 99.9 & 99.4 & 99.7 & 99.7 & 99.3 & 99.9 & 0 & 99.8 & 0 & 99.8 & 99.6 & \\
\hline
\end{tabular}




\begin{tabular}{|c|c|c|c|}
\hline $\begin{array}{l}\text { Site } \\
\text { Core, section } \\
\text { Interval }(\mathrm{cm})\end{array}$ & & $\begin{array}{l}791 \mathrm{~A} \\
2 \mathrm{H}-5 \\
74-76\end{array}$ & $\begin{array}{c}791 \mathrm{~A} \\
4 \mathrm{H}-5 \\
99-101\end{array}$ \\
\hline $\begin{array}{l}\text { Cumulative } \\
\text { volume percentages }\end{array}$ & $\begin{array}{c}\text { Class interval } \\
\begin{array}{c}(\phi) \\
1.75-\end{array} \\
2.00-1.75 \\
2.25-2.00 \\
2.50-2.25 \\
2.75-2.50 \\
3.00-2.75 \\
3.25-3.00 \\
3.50-3.25 \\
3.75-3.50 \\
4.00-3.75 \\
4.25-4.00 \\
4.50-4.25 \\
4.75-4.50 \\
5.00-4.75 \\
5.00-6.00 \\
7.00-6.00\end{array}$ & $\begin{array}{c}0 \\
0 \\
0 \\
0.6 \\
3 \\
8 \\
20.3 \\
35.5 \\
53.4 \\
68 \\
79.4 \\
87.9 \\
93.2 \\
94.8 \\
97.3 \\
99.8\end{array}$ & $\begin{array}{c}0 \\
0 \\
0 \\
0 \\
0.6 \\
2 \\
9.6 \\
20.5 \\
40.3 \\
57.8 \\
73.1 \\
84.7 \\
92.1 \\
94.3 \\
97 \\
99.6\end{array}$ \\
\hline $\begin{array}{l}\text { Site } \\
\text { Core, section } \\
\text { Interval }(\mathrm{cm})\end{array}$ & & $\begin{array}{l}791 \mathrm{~A} \\
39 X-1 \\
90-92\end{array}$ & $\begin{array}{l}791 A \\
42 X-1 \\
31-33\end{array}$ \\
\hline $\begin{array}{c}\text { Cumulative } \\
\text { volume percentages }\end{array}$ & $\begin{array}{c}\text { Class interval } \\
(\phi) \\
1.75- \\
2.00-1.75 \\
2.25-2.00 \\
2.50-2.25 \\
2.75-2.50 \\
3.00-2.75 \\
3.25-3.00 \\
3.50-3.25 \\
3.75-3.50 \\
4.00-3.75 \\
4.25-4.00 \\
4.50-4.25 \\
4.75-4.50 \\
5.00-4.75 \\
5.00-6.00 \\
7.00-6.00\end{array}$ & $\begin{array}{c}0 \\
0 \\
0 \\
1.4 \\
5.6 \\
13.8 \\
27.6 \\
43.4 \\
59.3 \\
71.8 \\
81 \\
87.9 \\
92.3 \\
93.8 \\
96.6 \\
99.4\end{array}$ & $\begin{array}{c}0 \\
0 \\
0 \\
1 \\
4.2 \\
10.7 \\
23.2 \\
38 \\
54.4 \\
67.6 \\
77.8 \\
85.6 \\
90.6 \\
92.4 \\
95.9 \\
99.1\end{array}$ \\
\hline
\end{tabular}


A

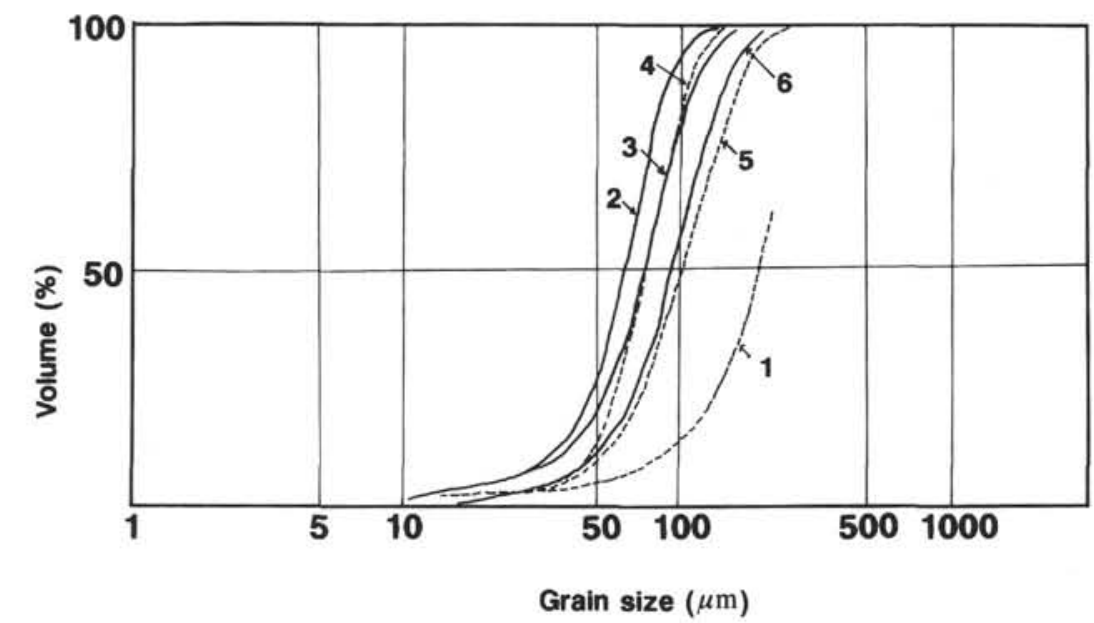

B

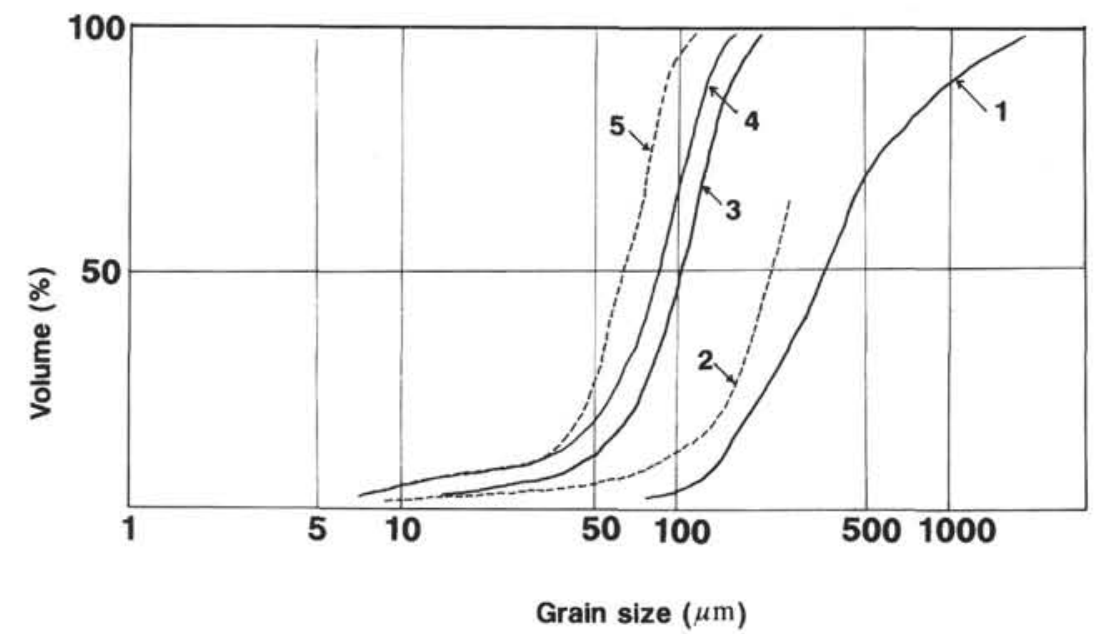

Figure 9. Cumulative grain-size distribution of the Quaternary volcanic ash layers. Grain sizes are indicated by microns $(\mu \mathrm{m})$ in this diagram. A. Forearc Site 792. Broken lines indicate black ash layers and solid lines indicate white ash layers. (1) Sample 126-792B-8X-3, 60-62 cm; (2) Sample 126-792A-1H-1, 38-40 cm; (3) Sample 126-792A-8H-3, 40-42 cm; (4) Sample 126-792A-5H-2, 141-143 cm; (5) Sample 126-792A-4H-5, 19-21 cm; (6) Sample 126-792A-9H-4, 6-8 cm. B. Backarc Site 790. Broken lines indicate black ash layers and solid lines indicate white ash layers. (1) Sample 126-790B-5H-5, 43-47 cm; (2) Sample 126-790C-14X-2, 135-138 cm; (3) Sample 126-790C-5H-5, 4-6 cm; (4) Sample 126-790B-7H-6, 146-148 cm; and (5) Sample 126-790B-8H-6, 84-86 cm.

1965; Letouzey and Kimura, 1985). These tephras are distributed partly onshore and, for the most part, in submarine areas around the Philippine Sea.

\section{ACKNOWLEDGMENTS}

During Leg 126, Captain Ribbon and the crew of the JOIDES Resolution helped us, sometimes beyond the call of duty. These are warmly acknowledged. B. Taylor, J. Gill, R. Hiscott, and K. Marsaglia gave us valuable discussion and a warm understanding of the ashy sediments.

Thanks also go to Drs. M. Yuasa, S. Nagaoka, N. Kotake, Y. Hirata, and $\mathrm{H}$. Matsuoka for their critical discussions on the tephras. Mrs. Kanehara and Miss K. Ikari and A. Koizumi helped us during the preparation of the manuscript.

\section{REFERENCES}

Brouxel, M., Lapierre, H., Michard, A., and Albarede, F., 1987. The deep layers of a Paleozoic arc: geochemistry of the Copley-Balaklala Series, northern California. Earth Planet. Sci. Lett., 85:386-400.

Brown, G., and Taylor, B., 1988. Sea-floor mapping of the Sumisu Rift, Izu-Ogasawara (Bonin) Island Arc. Bull. Geol. Surv. Jpn., 39:23-38.

Christiansen, R. L., and Lipman, P. W., 1972. Cenozoic volcanism and platetectonic evolution of the western United States. II. Late Cenozoic. Philos. Trans. R. Soc. London, Ser. A, 271:249-284.

Eaton, G. P., 1982. The Basin and Range Province: origin and tectonic significance. Annu. Rev. Earth Planet. Sci., 10:409-440.

1984. The Miocene Great Basin of western North America as an extending back-arc region. Tectonophysics, 102:275-295.

Fisher, R. V., 1984. Submarine volcaniclastic rocks. In Kokelaar, B. P., and Howells, M. F. (Eds.), Marginal Basin Geology: Volcanic and Associated 

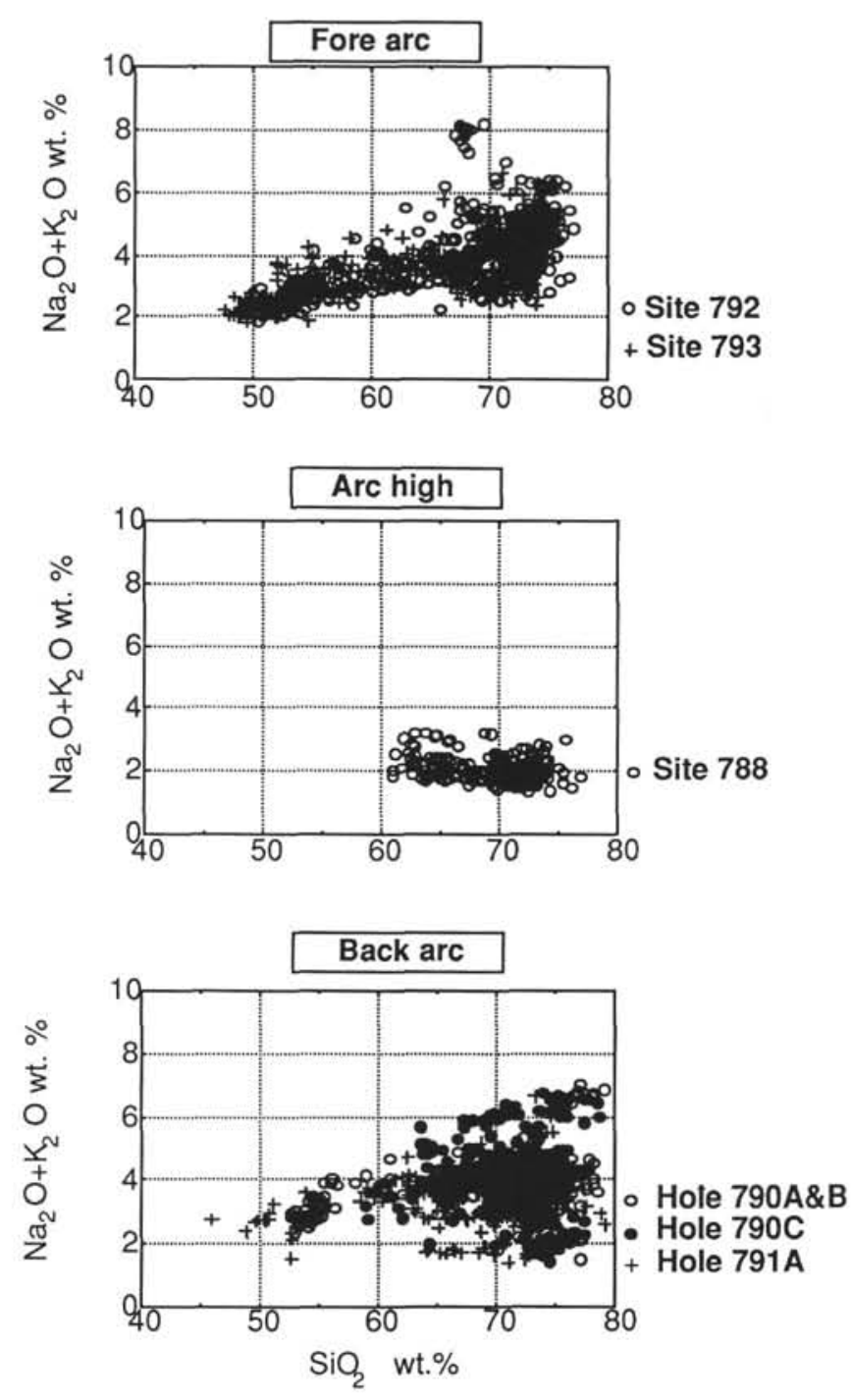

Figure $10 . \mathrm{SiO}_{2}$-alkali diagram of the Quaternary volcanic ash layers at forearc Sites 792 and 793, arc Site 788, and backarc Sites 790/791.

Sedimentary Processes in Modern and Ancient Basins. Spec. Publ., Geol. Soc. London, 16:5-28.

Fiske, R. S., 1986. Shallow marine volcanic field in the Mio-Pliocene Shirahama Group, Izu Peninsula, Japan. Geol. Soc. Am. Abstr. Programs, 1986 Annual Meeting, 602.

Fiske, R. S., and Cashman, K. V., 1989. Submarine fallout deposit in the Shirahama Group (Mio-Pliocene), Japan. Abstr. Vol., Int. Assoc. Volcanol. Chem. Earth's Interior, Gen. Assem., Santa Fe, New Mexico, No. 92.

Fujioka, K., 1983a. History of the explosive volcanism of the Tohoku Arc from the core sediment samples of the Japan Trench. J. Volcanol. Soc. Jpn., 28:41-58.

_ 1983b. Where were the "Kuroko deposits" formed, looking for the present day analogy. Min. Geol. (Tokyo), Spec. Vol., 11:55-68.

, 1988. A possible nascent rift in northern Izu-Ogasawara arc. In Tectonics of Eastern Asia and Western Pacific Continental Margin. 1988 DELP Tokyo Int. Symp. and Sixth Japan-U.S.S.R. Geotectonic Symp., 25. (Abstract)

Fujioka, K., Furuta, T., and Arai, F., 1980. Petrography and geochemistry of volcanic glass: Leg 57, Deep Sea Drilling Project. In von Huene, R., Nasu, N., et al., Init. Repts. DSDP, 56, 57, Pt. 2: Washington (U.S. Govt. Printing Office), 1049-1066.

Fujioka, K., Nishimura, A., Koyama, M., and Kotake, N., 1990. Bimodal arc volcanism and backarc rifting along Izu-Bonin Arc. Eos, 71:956.
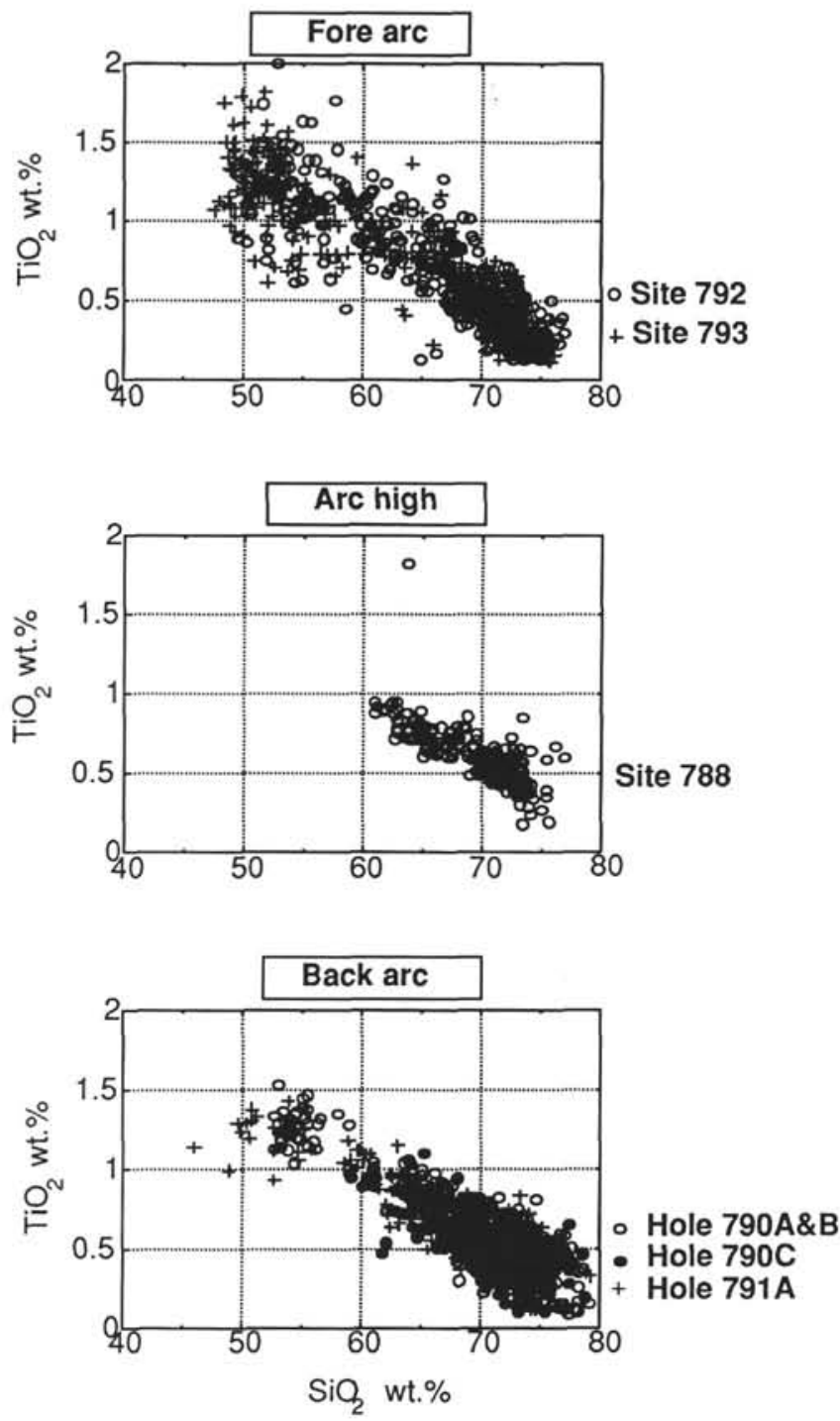

Figure 11. $\mathrm{SiO}_{2}-\mathrm{TiO}_{2}$ diagram of the Quaternary tephras at forearc Sites 792 and 793, arc Site 788, and backarc Sites 790/791.

Furuta, T., and Arai, F., 1980a. Petrographic and geochemical properties of tephras in Deep Sea Drilling Project cores from the north Philippine Sea. In Klein, G. de V., Kobayashi, K., et al., Init. Repts. DSDP, 58: Washington (U.S. Govt. Printing Office), 617-627.

, 1980b. Petrographic properties of tephras, Leg 56, Deep Sea Drilling Project. In von Huene, R., Nasu, N., et al., Init. Repts. DSDP, 56, 57, Pt. 2: Washington (U.S. Govt. Printing Office), 1043-1048.

Furuta, T., Fujioka, K., and Arai, F., 1986. Widespread submarine tephra around Japan, petrographic and chemical property. Mar. Geol., 72:125-146.

Gill, J. B., 1981. Orogenic Andesites and Plate Tectonics: New York (Springer-Verlag).

Gill, J. B., Stork, A. L., and Whelen, P. M., 1984. Volcanism accompanying backarc basin development in the Southwest Pacific. Tectonophysics, 102:207-224.

Gill, J., Torssander, P., Lapierre, H., Taylor, R., Kaiho, K., Koyama, M., Kusakabe, M., Aitchison, J., Cisowsky, S., Dadey, K., Fujioka, K., Klaus, A., Lovell, M., Marsaglia, K., Pezard, P., Taylor, B., and Tazaki, K., 1990. Explosive deep water basalt in the Sumisu backarc rift. Science, 248:1214-1217.

Hawkins, J. W., and Melchior, J. T., 1985. Petrology of Mariana Trough and Lau Basin basalts. J. Geophys. Res., 90:11431-11468.

Hochstaedter, A. G., Gill, J. B., Kusakabe, M., Newman, S., Pringle, M., Taylor, B., and Fryer, P., 1990a. Volcanism in the Sumisu Rift. I. Major 

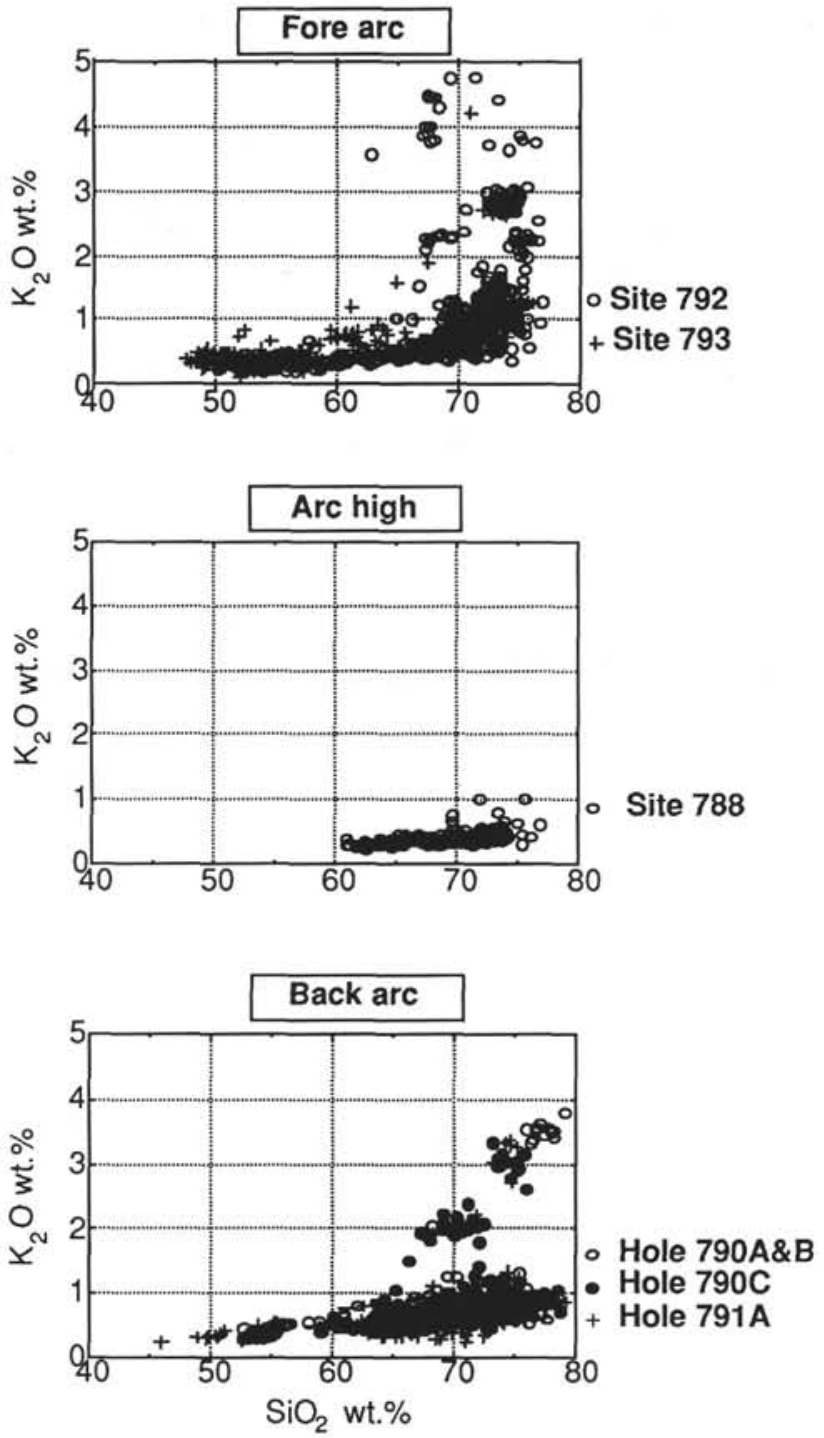

Figure 12. $\mathrm{SiO}_{2}-\mathrm{K}_{2} \mathrm{O}$ diagram of the Quaternary tephras at forearc Sites 792 and 793, arc Site 788, and backarc Sites 790/791.

element, volatile, and stable isotope geochemistry. Earth Planet. Sci. Lett., 100:179-194.

Hochstaedter, A. G., Gill, J. B., and Morris, J. D., 1990b. Volcanism in the Sumisu Rift. II. Subduction and non-subduction related components. Earth Planet. Sci. Lett., 100:195-209.

Ikeda, Y., and Yuasa, M., 1989. Volcanism in nascent back-arc basins behind the Shichito Ridge and adjacent areas in the Izu-Ogasawara arc, northwest Pacific: evidence for mixing between E-type MORB and island arc magmas at the initiation of back-arc rifting. Contrib. Mineral. Petrol., 101:377-393.

Inmann, D. L., 1952. Measures of describing the size distribution of sediments. J. Sediment. Petrol., 22:125-145.

Kennett, J. P., MacBirney, A. R., and Thunell, R. C., 1979. Episodes of Cenozoic volcanism in the circum-Pacific region. J. Volcanol. Geotherm. Res., 2:145-163.
Table 4. Chemistry of volcanic glass shards, Sites 788, 790/791, and 792/793.

\begin{tabular}{lccccc}
\hline Site & $\mathrm{SiO}_{2}$ & $\mathrm{TiO}_{2}$ & $\mathrm{~K}_{2} \mathrm{O}$ & $\mathrm{Na}_{2} \mathrm{O}$ & $\begin{array}{c}\text { Total } \\
\text { alkali }\end{array}$ \\
\hline $790 / 791$ & $65 \%-80 \%$ & $0.1 \%-1.0 \%$ & $0.5 \%-4.0 \%$ & $1.0 \%-6.0 \%$ & $1.7 \%-7.0 \%$ \\
788 & $70 \%-80 \%$ & $0.3 \%-1.0 \%$ & $0.2 \%-0.7 \%$ & $1.3 \%-2.7 \%$ & $1.7 \%-3.0 \%$ \\
$792 / 793$ & $65 \%-80 \%$ & $0.1 \%-1.0 \%$ & $0.2 \%-4.5 \%$ & $1.6 \%-4.7 \%$ & $2.5 \%-8.4 \%$
\end{tabular}

Kokelaar, B. P., Bevins, R. E., and Roach, R. A., 1985. Submarine silicic volcanism and associated sedimentary and tectonic processes, Ramsey Islands, SW Wales. Geol. Soc, London, 142:591-613.

Konishi, K., 1965. Geotectonic framework of the Ryukyu Islands (Nanseishoto). J. Geol. Soc. Jpn., 71:437-457.

Kotake, N., 1988. Upper Cenozoic marine sediments in southern part of the Boso Peninsula, central Japan. J. Geol. Soc. Jpn., 94:187-206.

Kuno, H., 1966. Lateral variation of basalt magma type across continental margins and island arcs. Bull. Volcanol., 19:195-222.

Leg 126 Scientific Drilling Party, 1989. ODP Leg 126 drills the Izu-Bonin Arc. Geotimes, 34:36-38.

Leg 126 Shipboard Scientific Party, 1989. Arc volcanism and rifting. Nature, 342:18-20.

Letouzey, J., and Kimura, M., 1985. Okinawa trough genesis: structure and evolution of a backarc basin developed in a continent. Mar. Pet. Geol., 2:111-130.

Machida, H., and Arai, F., 1983. Extensive ash falls in and around the sea of Japan from large late Quaternary eruptions. J. Volcanol. Geotherm. Res., 18:151-164.

1988. A review of late Quaternary deep-sea tephras around Japan. Quart. Res., 26:227-242.

Miyashiro, A., 1974. Volcanic rock series in island arcs and active continental margins. Am. J. Sci., 274:321-355.

Murakami, F., 1988. Structural framework of the Sumisu Rift, Izu-Ogasawara Arc. Bull. Geol. Surv. Jpn., 39:1-21.

Nakamura, K., 1964. Volcano-stratigraphic study of Oshima Volcano, Izu. Bull. Earthquake Res. Inst., Univ. Tokyo, 42:649-728.

Nishimura, A., and Murakami, F., 1988. Sedimentation of the Sumisu Rift, Izu-Ogasawara Arc. Bull. Geol. Surv. Jpn., 39:39-61.

Omura, A., 1984. Radio-isotope of uranium and thorium. Earth Monthly, 6:523-528.

Reagan, M. K., and Meijer, D., 1984. Geology and geochemistry of early sea-volcanic rocks from Guam. Bull. Geol. Soc. Am., 95:701-713.

Sparks, R.S.J., Wilson, L., and Sigurdsson, H., 1981. The pyroclastic deposits of the 1875 eruption of Askja, Iceland. Philos. Trans. R. Soc. London, Ser. A, 299:241-273.

Takada, A., Oshima, O., Aramaki, S., Ono, K., Yoshida, T., and Kajima, K., in press. Geology of Aogashima Volcano, Izu Islands, Japan. Bull. Volcanol. Soc. Jpn.

Taylor, B., Fujioka, K., et al., 1990. Proc. ODP, Init. Repts., 126: College Station, TX (Ocean Drilling Program).

Walker, G.P.L., 1971. Grain size characteristics of pyroclastic deposits. $J$. Geol., 79:696-714.

Yamazaki, T., 1988. Heat flow in the Sumisu Rift, Izu-Ogasawara (Bonin) Arc. Bull. Geol. Surv. Jpn., 39:63-70.

Yokoyama, T., Danhara, T., and Yamashita, T., 1986. A new refractometer for volcanic glass. J. Volcanol. Soc. Jpn., 25:21-30.

Date of initial receipt: 2 January 1991

Date of acceptance: 22 October 1991

Ms 126B-116 
Table 5. Correlation of marine tephras by mineral assemblage, chemistry, glass type, refractive indices, and age of the volcanic ash layers.

\begin{tabular}{|c|c|c|c|c|c|c|}
\hline Core, section, interval $(\mathrm{cm})$ & 1) & $792 \mathrm{~A}-3 \mathrm{H}-1, \quad 86-88$ & $793 \mathrm{~A}-4 \mathrm{H}-2, \quad 96-98$ & 2) & $792 \mathrm{~A}-5 \mathrm{H}-3,131-133$ & $442 A-5-3,30-34^{*}$ \\
\hline Age & & CN14b & CN14b-a & & $\mathrm{CN} 14 \mathrm{a}$ & p.lacunosa \\
\hline R.I.(Mean) & & 1.5067 & 1.5067 & & 1.5013 & $1.501-1.504$ \\
\hline R.I.(Range) & & $1.5058-1.5080$ & $1.504-1.5047$ & & $1.4998-1.5024$ & $1.500-1.505$ \\
\hline Mineral assemblage & & $\mathrm{pl}++, \mathrm{hb}+$ & $\mathrm{pl}++, \mathrm{hb}+$ & & $\mathrm{pl}++, \mathrm{cpx}+, \mathrm{hb}+, \mathrm{op}+$ & $\mathrm{pl}+, \mathrm{hb}$ \\
\hline Glass shape & & bw & bw & & bw & \\
\hline Thickness $(\mathrm{cm})$ & & $14(\mathrm{ML})$ & Dispersed in mud & & $90(\mathrm{ML})$ & \\
\hline Glass grain size(Max) & & 0.25 & 0.15 & & 0.2 & 0.2 \\
\hline Mode & & $95 / 5 / 0$ & $94 / 5 / 1$ & & $95 / 5 / 0$ & \\
\hline
\end{tabular}

\begin{tabular}{|c|c|c|c|c|c|c|}
\hline Core,section, interval (cm) & 3) & $792 \mathrm{~B}-1 \mathrm{H}-1,50-52$ & Kurasaki volcano & 4) & $792 \mathrm{~A}-1 \mathrm{H}-5,81-83$ & Aso $-4^{* *}$ \\
\hline Age & & CN15 & & & CN15 & 80,000 B.P. \\
\hline R.I.(mean) & & 1.5065 & & & 1.5096 & \\
\hline R.I.(range) & & $1.5056-1.5073$ & & & $1.509-1.5108$ & $1.506-1.514$ \\
\hline Mineral assemblage & & $\mathrm{pl}++$ & & & $\mathrm{pl}++, \mathrm{cpx}+, \mathrm{hb}+, \mathrm{op}+$ & $\mathrm{pl}, \mathrm{hb}, \mathrm{opx}, \mathrm{cpx}$ \\
\hline Glass shape & & bw & & & bw & bw $\gg$ pum \\
\hline Thickness $(\mathrm{cm})$ & & 7 & & & 3 & \\
\hline Glass grain size(Max) & & 0.05 & & & 0.55 & \\
\hline Mode & & & & & $93 / 5 / 2$ & \\
\hline
\end{tabular}



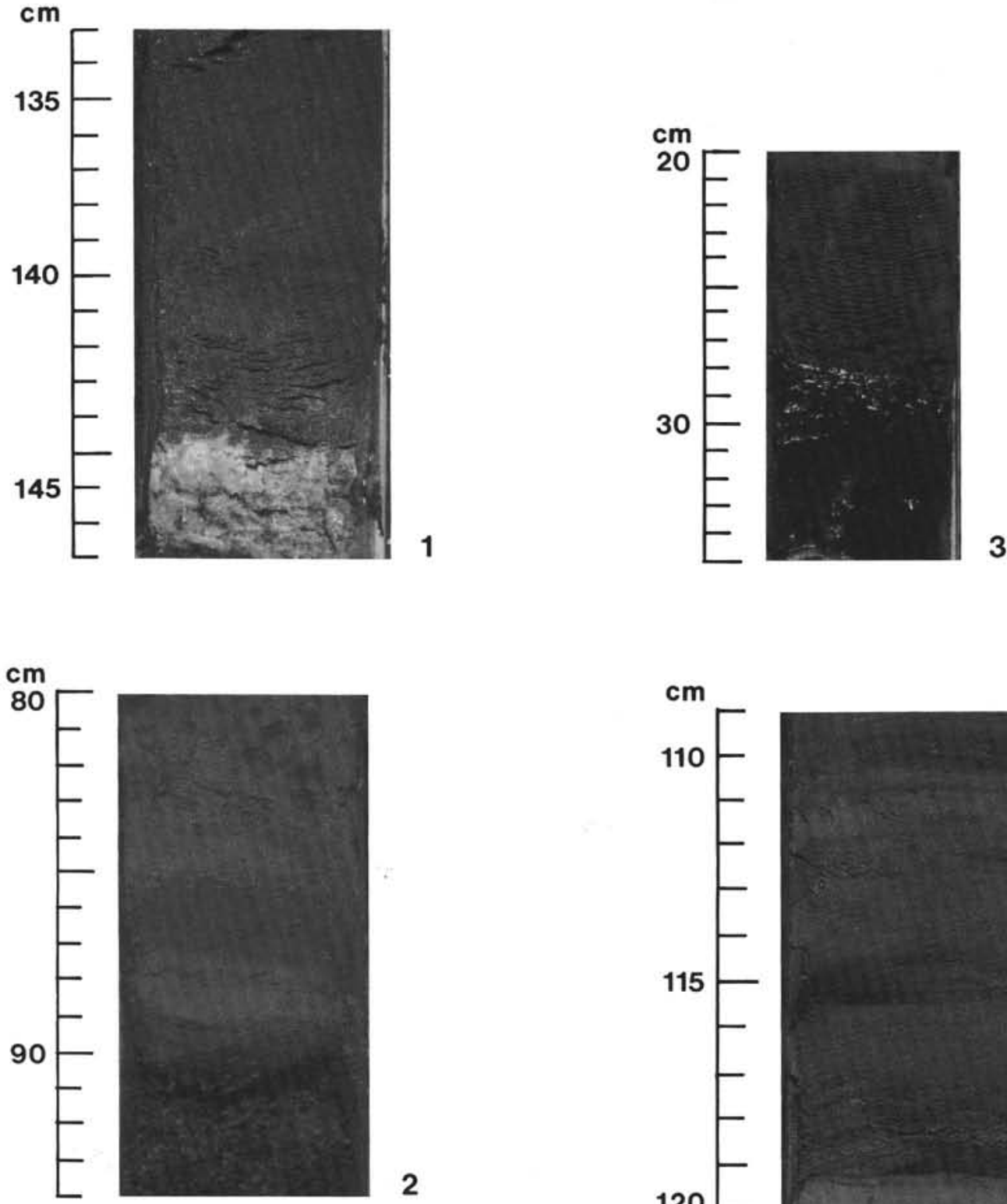

2

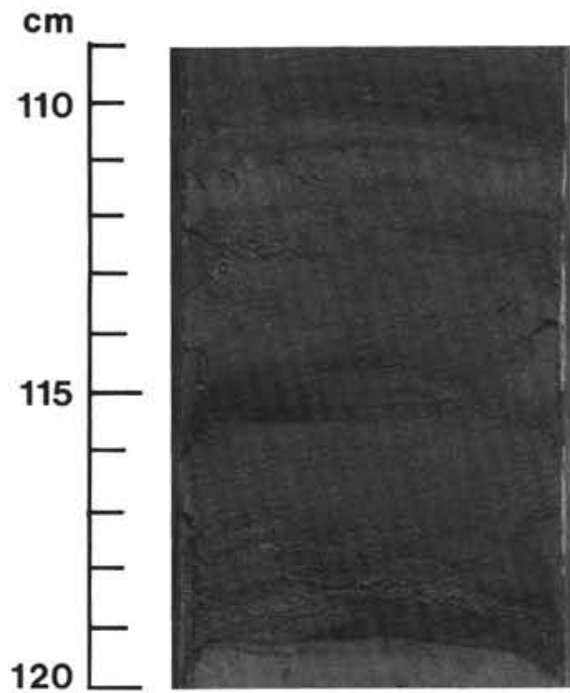

Plate 1. Core photographs. 1. Sample 126-790B-7H-6, 145-148 cm, simple white volcanic ash layer. 2. Section 126-792A-3H-1, $80-94 \mathrm{~cm}$, multiple ash layers with crystal-rich, coarse black ash at the bottom and $10 \mathrm{~cm}$ of thick, gray, mixed-ash layer above the black ash. 3. Section 126-790C-5H-5, 20-35 cm. multiple black ash layers with fine, sandy ash in the bottom of the tripartite (Taylor, Fujioka, et al., 1990). 4. Section 126-793A-7H-2, 109-120 cm, sharp-based, parallel-laminated, mixed volcanic-ash layer with notable grading structure inside (Taylor, Fujioka, et al., 1990). 


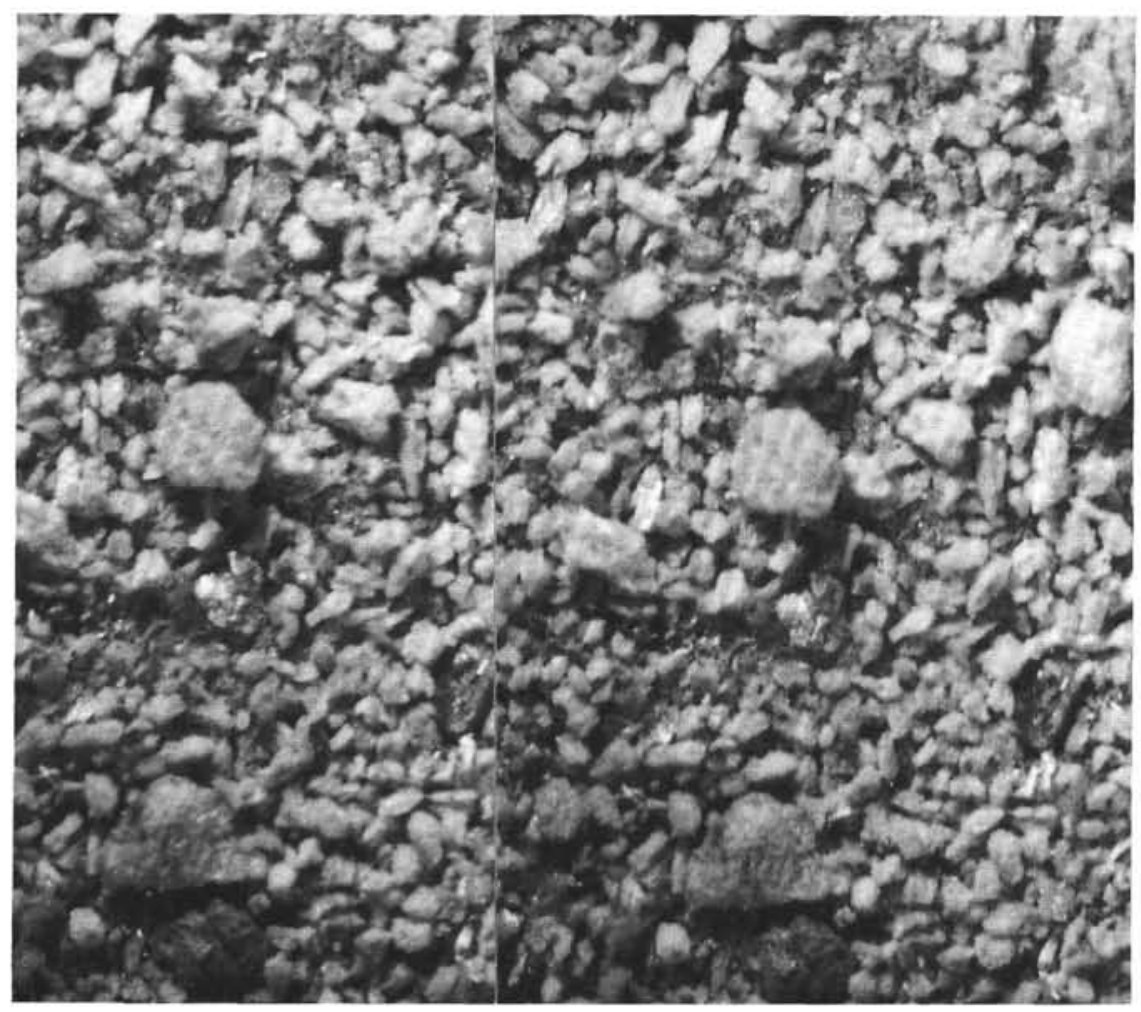

1

$1 \mathrm{~mm}$

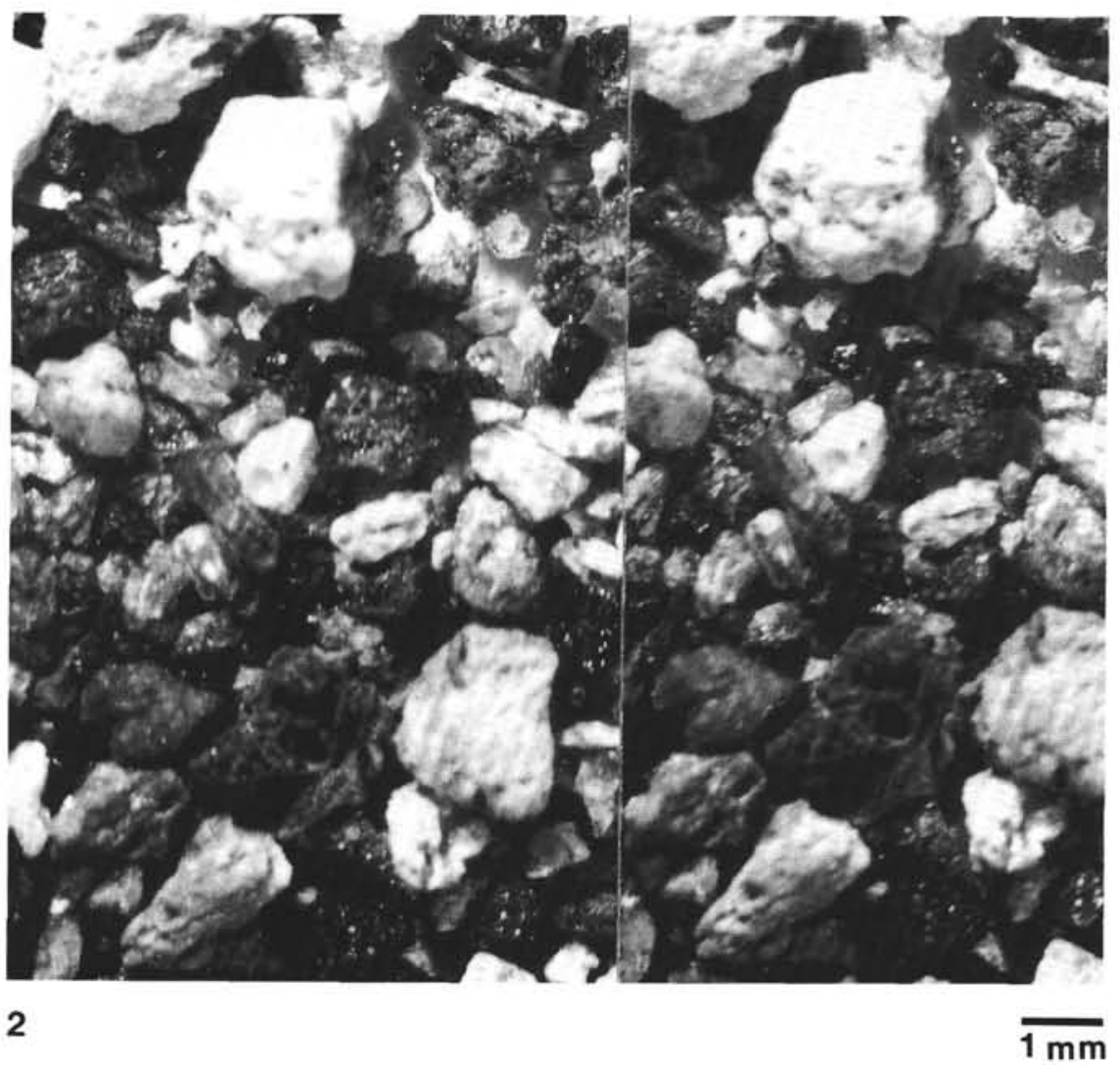

Plate 2. Stereographic photographs of the volcanic ash layers. 1. Sample 126-791A-10H-5, 117-119 $\mathrm{cm}$, example of poorly sorted coarse white pumice. 2. Sample $126-793 \mathrm{~A}-1 \mathrm{H}-2,118-120 \mathrm{~cm}$, example of mixed volcanic glass shards, consisting of white and dark gray pumices and scoria. 

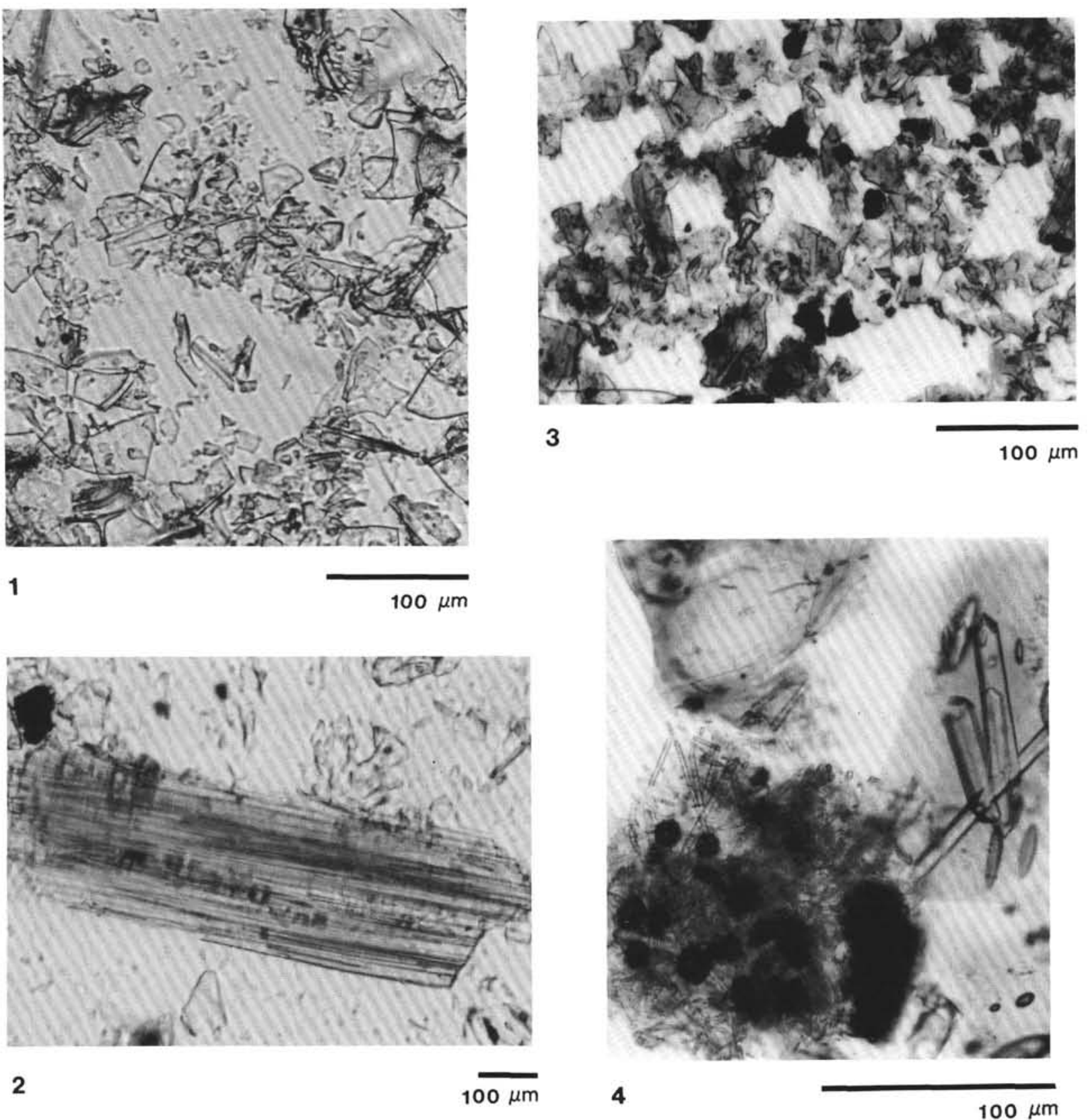

Plate 3. Photomicrographs of smear slides of the volcanic ash layer. 1. Sample 126-793A-7H-3, 35-37 cm, fine, white, bubble-wall-type glass shards. 2. Sample 126-792A-1H-6, 119-121 cm, tabular volcanic glass shards in the coarse, white tephra. 3. Sample 126-793A-8H-5, 28-30 $\mathrm{cm}$, brown glass in the fine, black tephra. 4. Sample 126-792A-6H-3, 130-132 cm, crystallized brown volcanic glass in the coarse black tephra. 